\title{
A study of the interplay between ionized gas and star clusters in the central region of NGC 5253 with 2D spectroscopy ${ }^{\star}$
}

\author{
A. Monreal-Ibero ${ }^{1}$, J. M. Vílchez ${ }^{2}$, J. R. Walsh ${ }^{1}$, and C. Muñoz-Tuñón ${ }^{3}$ \\ ${ }^{1}$ European Organisation for Astronomical Research in the Southern Hemisphere, Karl-Schwarzschild-Strasse 2, \\ 85748 Garching bei München, Germany \\ e-mail: [amonreal; jwalsh]@eso.org \\ 2 Instituto de Astrofísica de Andalucía (CSIC), C/ Camino Bajo de Huétor 50, 18008 Granada, Spain \\ e-mail: jvm@iaa.es \\ 3 Instituto de Astrofísica de Canarias, C/ vía Láctea, s/n, 38205 La Laguna, Spain \\ e-mail: cmt@iac.es
}

Received 28 January 2010 / Accepted 24 March 2010

ABSTRACT

\begin{abstract}
Context. Starbursts are one of the main contributors to the chemical enrichment of the interstellar medium. However, mechanisms governing the interaction between the recent star formation and the surrounding gas are not fully understood. Because of their a priori simplicity, the subgroup of $\mathrm{H}$ II galaxies constitute an ideal sample to study these mechanisms.

Aims. A detailed 2D study of the central region of NGC 5253 has been performed to characterize the stellar and ionized gas structure as well as the extinction distribution, physical properties and kinematics of the ionized gas in the central $\sim 210 \mathrm{pc} \times 130 \mathrm{pc}$.

Methods. We utilized optical integral field spectroscopy (IFS) data obtained with FLAMES.

Results. A detailed extinction map for the ionized gas in NGC 5253 shows that the largest extinction is associated with the prominent Giant H II region. There is an offset of $\sim 0$ ' 5 between the peak of the optical continuum and the extinction peak in agreement with findings in the infrared. We found that stars suffer less extinction than gas by a factor of $\sim 0.33$. The [S II $] \lambda 6717 /[\mathrm{S}$ II $] \lambda 6731$ map shows an electron density $\left(N_{\mathrm{e}}\right)$ gradient declining from the peak of emission in $\mathrm{H} \alpha\left(790 \mathrm{~cm}^{-3}\right)$ outwards, while the argon line ratio traces areas with $N_{\mathrm{e}} \sim 4200-6200 \mathrm{~cm}^{-3}$. The area polluted with extra nitrogen, as deduced from the excess [N II] $\lambda 6584 / \mathrm{H} \alpha$, extends up to distances of 3". $3(\sim 60 \mathrm{pc})$ from the maximum pollution, which is offset by $\sim 1$ ". 5 from the peak of continuum emission. Wolf-Rayet features are distributed in an irregular pattern over a larger area $(\sim 100 \mathrm{pc} \times 100 \mathrm{pc})$ and associated with young stellar clusters. We measured $\mathrm{He}^{+}$abundances over most of the field of view and values of $\mathrm{He}^{++} / \mathrm{H}^{+} \lessgtr 0.0005$ in localized areas which do not coincide, in general, with the areas presenting W-R emission or extra nitrogen. The line profiles are complex. Up to three emission components were needed to reproduce them. One of them, associated with the giant H II region, presents supersonic widths and [N II] $\lambda 6584$ and [S II] $\lambda \lambda 6717,6731$ emission lines shifted up to $40 \mathrm{~km} \mathrm{~s}^{-1}$ with respect to $\mathrm{H} \alpha$. Similarly, one of the narrow components presents offsets in the [N II] $\lambda 6584$ line of $\$ 20 \mathrm{~km} \mathrm{~s}^{-1}$. This is the first time that maps with such velocity offsets for a starburst galaxy have been presented. The observables in the giant H II region fit with a scenario where the two super stellar clusters (SSCs) produce an outflow that encounters the previously quiescent gas. The south-west part of the FLAMES IFU field is consistent with a more evolved stage where the star clusters have already cleared out their local environment.
\end{abstract}

Key words. galaxies: starburst - galaxies: dwarf - galaxies: individual: NGC 5253 - galaxies: ISM - galaxies: abundances galaxies: kinematics and dynamics

\section{Introduction}

Starbursts are events characterized by star-formation rates much higher than those found in gas-rich normal galaxies. They are considered one of the main contributors to the chemical enrichment of the interstellar medium (ISM) and can be found in galaxies covering a wide range of masses, luminosities, metallicities and interaction stages such as blue compact dwarfs, nuclei of spiral galaxies, or (Ultra)luminous Infrared Galaxies (see Conti et al. 2008, and references therein).

A particularly interesting subset are the H II galaxies, identified for the first time by Haro (1956): gas-rich, metal poor $\left(1 / 40 Z_{\odot} \lesssim Z \lesssim 1 / 3 Z_{\odot}\right)$ dwarf systems characterized by the presence of large ionized H II regions that dominate their optical spectra (see Kunth \& Östlin 2000, for a review of these galaxies).

* Based on observations collected at the European Organisation for Astronomical Research in the Southern Hemisphere, Chile (ESO Programme 078.B-0043).
These systems are a priori simple, which makes them the ideal laboratories to test the interplay between massive star formation and the ISM.

NGC 5253, an irregular galaxy located in the Centarus A/M 83 galaxy complex (Karachentsev et al. 2007), is a local example of an H II galaxy. This galaxy is suffering a burst of star formation which is believed to have been triggered by an encounter with M 83 (van den Bergh 1980). This is supported by the existence of the H I plume extending along the optical minor axis which is best explained as tidal debris (Kobulnicky \& Skillman 2008).

NGC 5253 constitutes an optimal target for the study of the starburst phenomenon. On the one hand, its proximity allows a linear spatial resolution to be achieved that is good enough to study the details of the interplay between the different components (i.e. gas, dust and star clusters) in the central region. On the other hand, this system has been observed in practically all spectral ranges from the X-ray to the radio, and therefore a large amount of ancillary information is available. 
Table 1. Basic data for NGC 5253.

\begin{tabular}{ccc}
\hline \hline Parameter & Value & Ref. \\
\hline Name & NGC 5253 & (a) \\
Other designations & ESO 445- G004, Haro 10 & (a) \\
RA $(J 2000.0)$ & $13 \mathrm{~h} 39 \mathrm{~m} 55.9 \mathrm{~s}$ & (a) \\
Dec $(\mathrm{J} 2000.0)$ & $-31 \mathrm{~d} 38 \mathrm{~m} 24 \mathrm{~s}$ & (a) \\
$z$ & 0.001358 & (a) \\
$D(\mathrm{Mpc})$ & 3.8 & (b) \\
scale $\left(\mathrm{pc} /{ }^{\prime \prime}\right)$ & 18.4 & \\
$m_{B}$ & 10.78 & (c) \\
$M_{B}$ & -17.13 & (c) \\
$U-B$ & -0.30 & (c) \\
$B-V$ & 0.50 & (c) \\
$V-R$ & 0.32 & (c) \\
$M_{\mathrm{HI}}\left(M_{\odot}\right)$ & $1.4 \times 10^{8} M_{\odot}$ & (d) \\
$Z / Z_{\odot}$ & $\sim 0.3^{(*)}$ & (e) \\
$\log \left(L_{\text {iri }} / L_{\odot}\right)$ & 8.95 & (f) \\
$\log \left(L_{\text {ir }} / L_{\odot}\right)$ & 9.21 & (f) \\
\hline
\end{tabular}

References. ${ }^{(*)}$ We assumed $12+\log (\mathrm{O} / \mathrm{H})_{\odot}=8.66$ (Asplund et al. 2004); (a) NASA/IPAC Extragalactic Database (NED); (b) Sakai et al. (2004); (c) Taylor et al. (2005); (d) Kobulnicky \& Skillman (2008); (e) Kobulnicky et al. (1999); ${ }^{(f)}$ Sanders et al. (2003). Re-scaled to the distance adopted here.

The basic characteristics of this galaxy are compiled in Table 1. Its stellar content has been widely studied and more than 300 stellar clusters have been detected (Cresci et al. 2005). Multi-band photometry with the WFPC2 has revealed that those in its central region present typical masses of $\sim 2-120 \times 10^{3} M_{\odot}$ and are very young, with ages of $\sim 1-12 \mathrm{Myr}$ (e.g. Harris et al. 2004). In particular, HST-NICMOS images have revealed that the nucleus of the galaxy is made out of two very massive $\left(\sim 1-2 \times 10^{6} M_{\odot}\right)$ super stellar clusters (SSCs), with ages of about $\sim 3.5 \mathrm{Myr}$, separated by $\sim 0$.' 4 (Alonso-Herrero et al. 2004), and which are coincident with the double radio nebula detected at $1.3 \mathrm{~cm}$ (Turner et al. 2000). Also, detection of spectral features characteristic of Wolf-Rayet (W-R) stars in specific regions of the galaxy have been reported (e.g. Schaerer et al. 1997). Recently, seven supernova remnants have been detected in the central region of this galaxy by means of the $[\mathrm{Fe}$ II $] \lambda 1.644 \mu \mathrm{m}$ emission (Labrie \& Pritchet 2006).

NGC 5253 presents a filamentary structure in $\mathrm{H} \alpha$ (e.g. Martin 1998) associated with extended diffuse emission in X-ray which can be explained as multiple superbubbles around its OBs associations and SSCs that are the results of the combined action of stellar winds and supernovae (Strickland \& Stevens 1999; Summers et al. 2004).

The measured metallicity of this galaxy is relatively low (see Table 1) and presents a generally uniform distribution. However, an increase in the abundance of nitrogen in the central region of 2-3 times the mean has been reported (Walsh \& Roy 1989; Kobulnicky et al. 1997; López-Sánchez et al. 2007). No other elemental species appears to present spatial abundance fluctuations. The reason for this nitrogen enhancement has not been fully clarified yet although a connection with the W-R population has been suggested.

On account of their irregular structure, a proper characterization of the physical properties of H II galaxies, necessary to explore the interplay of mechanisms acting between gas and stars, requires high quality two-dimensional spectral information able to produce a continuous mapping of the relevant quantities. Such observations have traditionally been done in the optical and nearinfrared by mapping the galaxy under study with a long-slit (e.g.
Vílchez \& Iglesias-Páramo 1998; Walsh \& Roy 1989). This is, however, expensive in terms of telescope time and might be affected by some technical problems such as misalignment of the slit or changes in the observing conditions with time. The advent and popularization of integral field spectroscopy (IFS) facilities, able to record simultaneously the spectra of an extended continuous field, overcomes these difficulties. Nevertheless, work based on this technique devoted to the study of H II galaxies is still relatively rare (e.g. Lagos et al. 2009; Bordalo et al. 2009; James et al. 2009; Kehrig et al. 2008; García-Lorenzo et al. 2008; Izotov et al. 2006).

Here, we present IFS observations of the central area of NGC 5253 in order to study the mechanisms that govern the interaction between the young stars and the surrounding ionized gas. The paper is organized as follows: Sect. 2 contains the observational and technical details regarding the data reduction and derivation of the required observables; Sect. 3 describes the stellar and ionized gas structure as well as the extinction distribution and the physical and kinematic properties of the ionized gas; Sect. 4 discusses the evolutionary stage of the gas surrounding the stellar clusters, focusing on the two most relevant areas of the field of view (f.o.v.). Section 5 itemises our results and conclusions.

\section{Observations, data reduction and line fitting}

\subsection{Observations}

Data were obtained with the Fibre Large Array Multi Element Spectrograph, FLAMES (Pasquini et al. 2002) at Kueyen, Telescope Unit 2 of the $8 \mathrm{~m}$ VLT at ESO's observatory on Paranal, on February 10, 2007. The central region of the galaxy was observed with the ARGUS Integral Field Unit (IFU) which has a field of view of $11^{\prime \prime} .5 \times 77^{\prime \prime} 3$ with a sampling of $0.52^{\prime \prime} /$ lens. In addition, ARGUS has 15 fibers that can simultaneously observe the sky and which were arranged forming a circle around the IFU. The precise covered area is shown in Fig. 1 which contains the FLAMES field of view over-plotted on an $\operatorname{HST} B, \mathrm{H} \alpha$, I colour image.

We utilized two different gratings in order to obtain information for the most important emission lines in the optical spectral range. Data were taken under photometric conditions and seeing ranged typically between $0{ }^{\prime} 8$ and $11^{\prime \prime} 0$. The covered spectral range, resolving power, exposure time and airmass for each configuration are shown in Table 2. In addition to the science frames, continuum and ThAr arc lamps exposures as well as frames for the spectrophotometric standard star CD-32 9927 were obtained.

\subsection{Data reduction}

The basic reduction steps for the FLAMES data were performed with a combination of the pipeline provided by ESO (version 1.0) $)^{1}$ via esorex, version 2.0.2 and some IRAF $^{2}$ routines. First of all we masked a bad column in the raw data using the task fixpix within IRAF. Then, each individual frame was processed using the ESO pipeline in order to perform bias subtraction, spectral tracing and extraction, wavelength calibration and correction of fibre transmission.

\footnotetext{
1 http://www . eso.org/projects/dfs/dfs-shared/web/vlt/ vlt-instrument-pipelines.html

2 The Image Reduction and Analysis Facility IRAF is distributed by the National Optical Astronomy Observatories which is operated by the association of Universities for Research in Astronomy, Inc. under cooperative agreement with the National Science Foundation.
} 


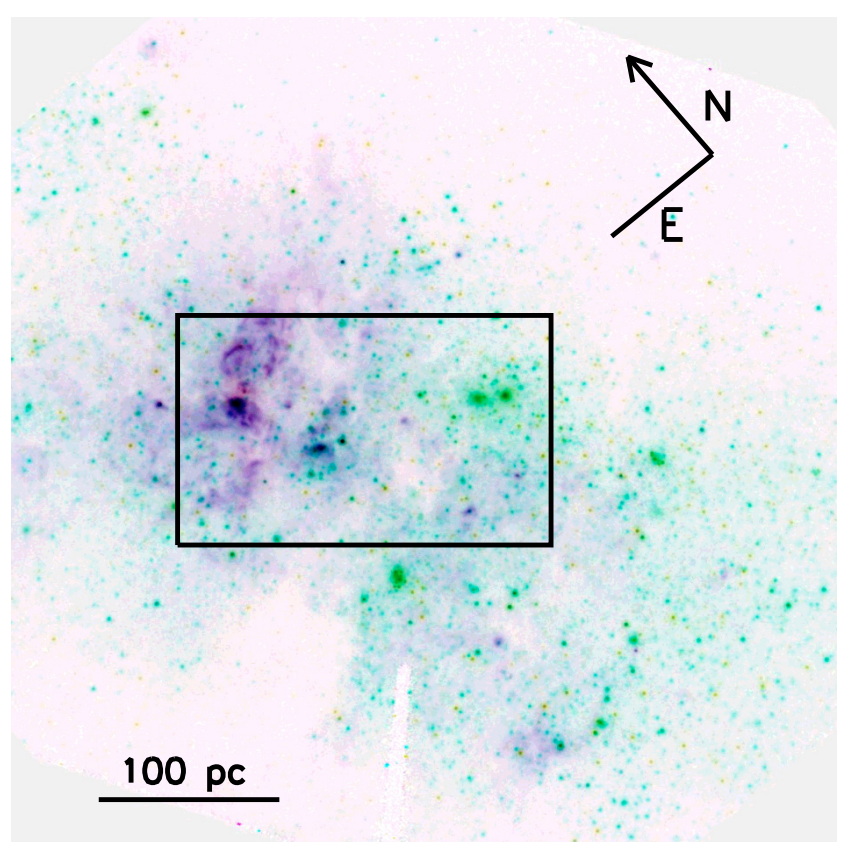

Fig. 1. False colour image in filters $F 435 W$ ( $B$, blue), $F 658 N$ (H $\alpha$, magenta), and $F 814 W$ ( $I$, green) for NGC 5253 using HST-ACS images (programme 10608, P.I.: Vacca) with the area covered by our FLAMES data over-plotted as a white rectangle. The orientation and scale for a distance of $3.8 \mathrm{Mpc}$ are indicated.

Table 2. Observation log.

\begin{tabular}{ccccc}
\hline \hline Grating & $\begin{array}{c}\text { Spectral range } \\
(\AA)\end{array}$ & Resolution & $\begin{array}{c}t_{\text {exp }} \\
(\mathrm{s})\end{array}$ & Airmass \\
\hline L682.2 & $6438-7184$ & 13700 & $5 \times 1500$ & $1.75-1.13$ \\
L479.7 & $4501-5078$ & 12000 & $5 \times 1500$ & $1.11-1.01$ \\
\hline
\end{tabular}

Uncertainties in the relative wavelength calibration were estimated by fitting a Gaussian to three isolated lines in every spectrum of the arc exposure. The standard deviation of the central wavelength for a certain line gives an idea of the associated error in that spectral range. We were able to determine the centroid of the lines with an uncertainty of $\sim 0.005 \AA$, which translates into velocities of $\sim 0.3 \mathrm{~km} \mathrm{~s}^{-1}$. The spectral resolution was very uniform over the whole field-of-view with values of $0.178 \pm 0.004 \AA$ and $0.241 \pm 0.009 \AA$, FWHM for the blue and red configuration respectively, which translates into $\sigma_{\text {instru }} \sim 4.7 \mathrm{~km} \mathrm{~s}^{-1}$.

For the sky subtraction, we created a good signal-to-noise $(\mathrm{S} / \mathrm{N})$ spectrum by averaging the spectra of the sky fibres in each individual frame. This sky spectrum was subsequently subtracted from every spectrum. In several of the sky fibres, the strongest emission lines, namely [O III] $\lambda 5007$ in the blue frames and $\mathrm{H} \alpha$ in the red frames, could clearly be detected. We attributed this effect to some cross-talk from the adjacent fibers. A direct comparison of the flux in the sky and adjacent fibers showed that this contribution was always $\$ 0.6 \%$ which is negligible in terms of sky subtraction. However, in order to reduce this contamination to a minimum, we decided not to use these fibres in the creation of the high $\mathrm{S} / \mathrm{N}$ sky spectra.

Regarding the flux calibration, a spectrum for the calibration star was created by co-adding all the fibers of the standard star frames. Then, a sensitivity function was determined with the IRAF tasks standard and sensfunc and science frames were calibrated with calibrate. Afterwards, frames corresponding to each configuration were combined and cosmic rays rejected with the task imcombine. As a last step, the data were reformatted into two easier-to-use data cubes, with two spatial and one spectral dimension, using the known position of the lenses within the array.

\subsection{Line fitting and map creation}

In order to obtain the relevant emission line information, line profiles were fitted using Gaussian functions. This procedure was done in a semi-automatic way using the IDL based routine MPFITEXPR $^{3}$ (Markwardt 2009) which offers ample flexibility in case constraints on the parameters of the fit are included, such as lines in fixed ratio. The procedure was as follows.

As a first step, we fit all the lines by a single Gaussian. The $\mathrm{H} \alpha+[\mathrm{N}$ II $]$ complex was fitted simultaneously by one Gaussian per emission line plus a flat continuum first-degree polynomial using a common width for the three lines and fixing the separation in wavelength between the lines according to the redshift provided at $\mathrm{NED}^{4}$ and the nitrogen line ratio $(\lambda 6583 / \lambda 6548)$ to 3 . The same procedure was repeated for the $[\mathrm{S}$ II $] \lambda \lambda 6717,6730$ doublet, the $[\mathrm{Ar}$ IV] $\lambda 4711$ line (which was fitted jointly with the [Fe III] $\lambda 4701$ and He I $\lambda 4713$ ) and the [ArIV] $\lambda 4740$ line (which was fitted jointly with the $[\mathrm{Fe}$ III] $] \lambda 4734$ line), but this time without any restriction on the line ratios. Finally, $\mathrm{H} \beta$, [O III $] \lambda 5007$, and $\mathrm{He} \mathrm{I} \lambda 6678$ were individually fitted.

This single Gaussian fit gave a good measurement for the line fluxes and the results of these fits are the used in all the forthcoming analysis, with the exception of the kinematics. This latter analysis requires a more complex line fitting scheme, since several lines showed signs of asymmetries and/or multiple components in their profiles for a large number of spaxels. In those cases, multi-component fits were performed. Over the whole field of view we compared the measured flux from performing the fit with a single Gaussian to the fit by several components in the brightest emission lines (namely: $\mathrm{H} \beta$, [O III $] \lambda 5007$, $\mathrm{H} \alpha,[\mathrm{N}$ II] $\lambda 6584$ and [S II] $\lambda \lambda 6717,6731)$. Differences between the two sets of line fluxes ranged typically from $0 \%$ to $15 \%$, depending on the spaxel and the emission line, and translated into differences in the line ratios $\lesssim 0.06 \mathrm{dex}$.

In all the cases, MPFITEXPR estimated an error for the fit using the standard deviation of the adjacent continuum. Those fits with a ratio between line flux and error less than three were automatically rejected. The remaining spectra were visually inspected and classified as good or bad fits.

Finally, for each of the observables, we used the derived quantity together with the position within the data-cube for each spaxel to create an image suitable to be manipulated with standard astronomical software. Hereafter, we will use both terms, map and image, when referring to these.

\section{Results}

\subsection{Stellar and ionized gas structure}

Figure 2 displays the stellar structure, as traced by a continuum close to $\mathrm{H} \alpha$, as well as the one for the ionized gas (traced by the $\mathrm{H} \alpha$ emission line). The over-plotted contours, which represent the HST-ACS images in the F659N and F814W bands convolved

\footnotetext{
3 See http://purl.com/net/mpfit

4 See http://nedwww.ipac. caltech.edu/
} 

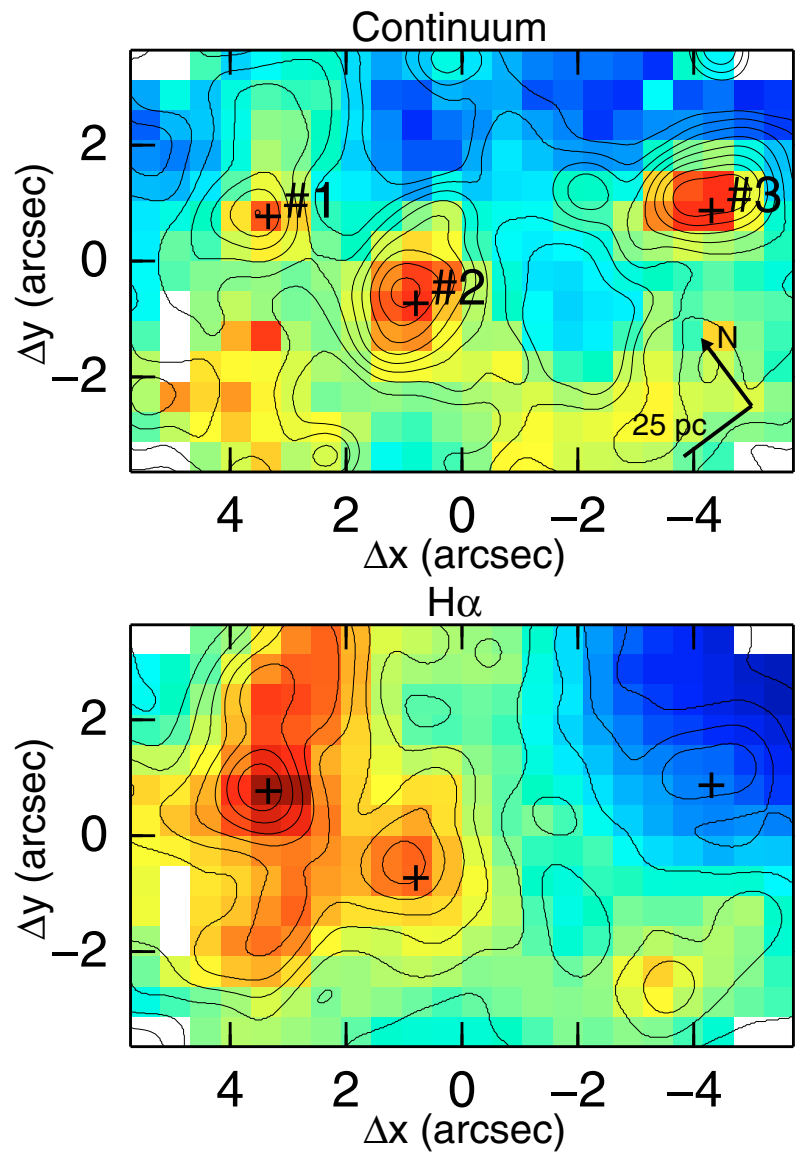

Fig. 2. Top: stellar component distribution as traced by a continuum map made from the average flux in the spectral range 6525-6545 and 6600-6620 ̊. Bottom: ionized gas distribution as traced by the $\mathrm{H} \alpha$ emission line. We have over-plotted contours corresponding to the HST-ACS images in the F814W (top) and F658N (bottom) filters (programme 10609, P.I.: Vacca) convolved with a Gaussian of 0.' 8 to simulate the seeing at Paranal. The position of the three main peaks of continuum emission are marked with crosses. Both maps are presented in logarithmic scale in order to emphasize the relevant morphological features and cover a range of 2.0 dex for the $\mathrm{H} \alpha$ and 0.9 dex for the continuum map. Flux units are arbitrary.

Table 3. Main reference clusters.

\begin{tabular}{cccccc}
\hline \hline \multirow{2}{*}{ Name } & FLAMES & \multicolumn{4}{c}{ Other names } \\
& coord.(" $\left.^{(\prime},{ }^{\prime \prime}\right)$ & C97 $^{(\mathrm{a})}$ & $\mathrm{H}^{(\mathrm{b})}$ & K97 $^{(\mathrm{c})}$ & AH04 $^{(\mathrm{d})}$ \\
\hline$\# 1$ & $(3.6,0.5)$ & $\mathrm{N} 5253-5$ & 1 & $\mathrm{UV3}$ & $\mathrm{C} 1+\mathrm{C} 2$ \\
$\# 2$ & $(1.0,-1.0)$ & $\mathrm{N} 5253-4$ & $4,8,24,25$ & $\mathrm{UV} 1$ & - \\
$\# 3$ & $(-4.2,1.0)$ & $\mathrm{N} 5253-3$ & 3,5 & - & $\mathrm{C} 4+\mathrm{C} 5$ \\
\hline
\end{tabular}

References. (a) Calzetti et al. (1997); (b) Harris et al. (2004); (c) Kobulnicky et al. (1997); (d) Alonso-Herrero et al. (2004).

with a Gaussian to match the seeing at Paranal, show good correspondence between the images created from the IFS data and the HST images (although obviously with poorer resolution for the ground-based FLAMES data). A direct comparison of these maps shows how the stellar and ionized gas structure differs.

The continuum image displays three main peaks of emission which will be used through the paper as reference. We have associated each of these peaks with one or more star clusters by direct comparison with ACS images. Table 3 compiles their positions within the FLAMES field of view together with the names of the corresponding clusters according to several reference works. These clusters trace a sequence in age as we move towards the right (south-west) in the FLAMES field of view. The clusters associated with peak \#1 are very young ( 3-8 Myr, Harris et al. 2004; Alonso-Herrero et al. 2004), those associated with peak \#2 display a range of ages from very young to intermediate age ( 6-170 Myr, Harris et al. 2004), while the stars in the pair of clusters associated with peak \#3 seem to have intermediate ages (70-113 Myr, Harris et al. 2004).

The $\mathrm{H} \alpha$ emission line reproduces the structure described by Calzetti et al. (1997) using an HST WFPC2 image. Briefly, the central region of NGC 5253 is divided into two parts by a dust lane that crosses the galaxy along the east-west direction (from $\sim\left[2{ }^{\prime \prime} 0,-33^{\prime \prime} 0\right]^{5}$ to the Complex \#3). Most of the $\mathrm{H} \alpha$ emission is located towards the north of this lane where there is giant $\mathrm{H}$ II region associated with the Complex \#1. This region shows two tongue-shaped extensions towards the upper and lower part of the FLAMES field of view (PA on the sky of $\sim 45^{\circ}$ and $\sim-135^{\circ}$, respectively) as well as a extension at $\mathrm{PA} \sim 155^{\circ}$ which contains the Complex \#2. Towards the south of the dust lane, the emission is dominated by a peak at $\sim\left[-3^{\prime \prime} .5,-2{ }^{\prime \prime} 5\right]$ which could be associated with cluster 17 in Harris et al. (2004).

\subsection{Extinction structure}

Extinction was derived assuming an intrinsic Balmer emission line ratio of $\mathrm{H} \alpha / \mathrm{H} \beta=2.87$ (Osterbrock \& Ferland 2006, for an $T_{\mathrm{e}}=10000 \mathrm{~K}$ ) and using the extinction curve of Fluks et al. (1994). Since the $\mathrm{H} \alpha$ and $\mathrm{H} \beta$ emission lines are separated by $\sim 1700 \AA$ and both of them had high signal-to-noise ratio in only a single exposure, we decided to obtain the extinction maps from the LR3 and LR6 exposures observed at the smallest airmass (1.009 and 1.160, respectively), thus minimizing any effect due to differential atmospheric refraction.

We have not included any correction for an underlying stellar population. We inspected carefully each individual spectrum to look for the presence of the stellar absorption feature in $\mathrm{H} \beta$. Only in those spaxels associated with the area around the Complex \#3 ( 9-10 spaxels or $\sim 1^{\prime \prime} .5 \times 1$ 1.'5 in total) was such an absorption detected. We estimated the influence of this component by fitting both the absorption and the emission component in the most affected spaxel. Equivalent widths were $\sim 3$ and $\sim 9 \AA$, respectively. In these spaxels, the absorption line was typically $\sim 15-20$ times wider and with about half - one third of the flux of the emission line. This implies an underestimation of $\mathrm{H} \beta$ emission line flux of about $10 \%$. For the particular area around Complex \#3, this translates into a real extinction of $A_{V} \sim 0.9$ mag instead of the measured $A_{V} \sim 1.2 \mathrm{mag}$.

The corresponding reddening map was determined assuming $E(B-V)=A_{V} / 3.1$ (Rieke \& Lebofsky 1985) and is presented in Fig. 3. This map shows that the extinction is distributed in a non-uniform manner ranging from $E(B-V)=0.16$ to 0.64 (mean 0.33, standard deviation, 0.07). Given that Galactic reddening for NGC 5253 is 0.056 (Schlegel et al. 1998), nearly all of the extinction can be considered intrinsic to the galaxy.

In general terms, the structure presented in this map coincides with the one presented by Calzetti et al. (1997). The dust lane mentioned in the previous section is clearly visible here and it causes extinction of $A_{V} \sim 0.10-1.13$ mag. However, the larger measured extinction values are associated with the giant $\mathrm{H}$ II region, in agreement with the $\mathrm{H}$ I distribution

\footnotetext{
5 Hereafter, the different quoted positions will be refereed as [", "] and using the FLAMES f.o.v. as reference.
} 


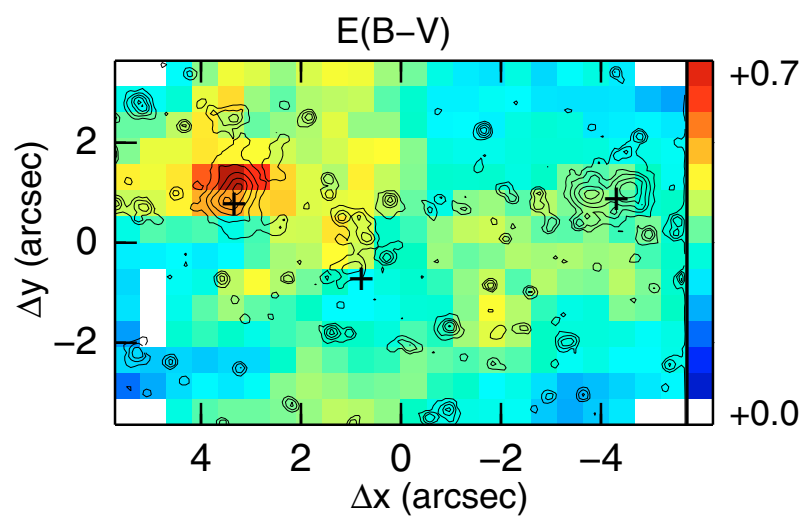

Fig. 3. Reddening map obtained from the hydrogen recombination lines assuming $\mathrm{H} \alpha / \mathrm{H} \beta=2.87$ and the extinction law of Fluks et al. (1994) and $E(B-V)=A_{V} / 3.1$ (Rieke \& Lebofsky 1985). The position of the three main peaks of continuum emission are shown for reference. We have over-plotted the F160W band NICMOS-HST image from Alonso-Herrero et al. (2002) with contours for comparison.

(Kobulnicky \& Skillman 2008). Dust in this area forms an S-shaped distribution with $A_{V} \sim 0.13-1.15 \mathrm{mag}$ in the arms.

In order to explore the relation between the extinction suffered by the gas and by the stellar populations, our $E(B-V)$ measurements were compared with colours defined ad hoc. For the covered spectral range, it is not possible to exactly simulate any of the existing standard filters. It is possible, however, to create filters relatively similar to the $g^{\prime}$ and $R_{\mathrm{c}}$ ones. We have simulated two set of filters.

In the first case, the flux was integrated over two large wavelength ranges $(465-495 \mathrm{~nm}$ and $643-673 \mathrm{~nm})$ in order to simulate broad filters. The relation between the reddening derived for the ionized gas and the derived colour, hereafter $\left(g_{1}^{\prime}-R_{\mathrm{c} 1}\right)$, is shown in the upper panel of Fig. 4, as would be observed with photometry. The first order polynomial fit to the data and the Pearson correlation coefficient are included on the plot. Also shown is the expected relation for an Im galaxy with foreground reddening. This latter was derived for the average of two Im templates (NGC 4449 and NGC 4485) from Kennicutt (1992) applying a foreground screen of dust with a standard Galactic reddening law (Cardelli et al. 1989) with $R=3.1$. There is a strong difference between the expected relation for a foreground screen of dust and the measured values. On the one hand, colours are much redder. On the other hand, the slope of the 1-degree polynomial fit is much less steep than the expected one. Also, there is a very good correlation between the $E(B-V)$ and our synthetic $\left(g^{\prime}-R_{\mathrm{c} 1}\right)$ colour. All this can be attributed to the contamination of the gas emission lines, mainly $\mathrm{H} \alpha$ and $\mathrm{H} \beta$, in our filters.

In the second set, we restricted the spectral ranges for the simulated filters to a narrower wavelength range which was free from the contamination of the main emission lines. The map for this line-free colour is displayed in Fig. 5. The structure resembles the one presented in Fig. 3 (i.e. dust lane, redder colours associated with the giant $\mathrm{H}$ II region), although there are differences, that can be attributed to differences in the properties of the stellar populations in the different clusters. The relation between the reddening and the corresponding $\left(g_{2}^{\prime}-R_{\mathrm{c} 2}\right)$ is shown in the lower panel of Fig. 4. This time colours are more similar to what is expected for a given stellar population suffering a certain amount of extinction. However, for a given colour, stars do not reach the expected reddening if gas and stars were suffering the same extinction (i.e. data points are below the green line). The ratio between the slopes indicates that extinction in the stars is
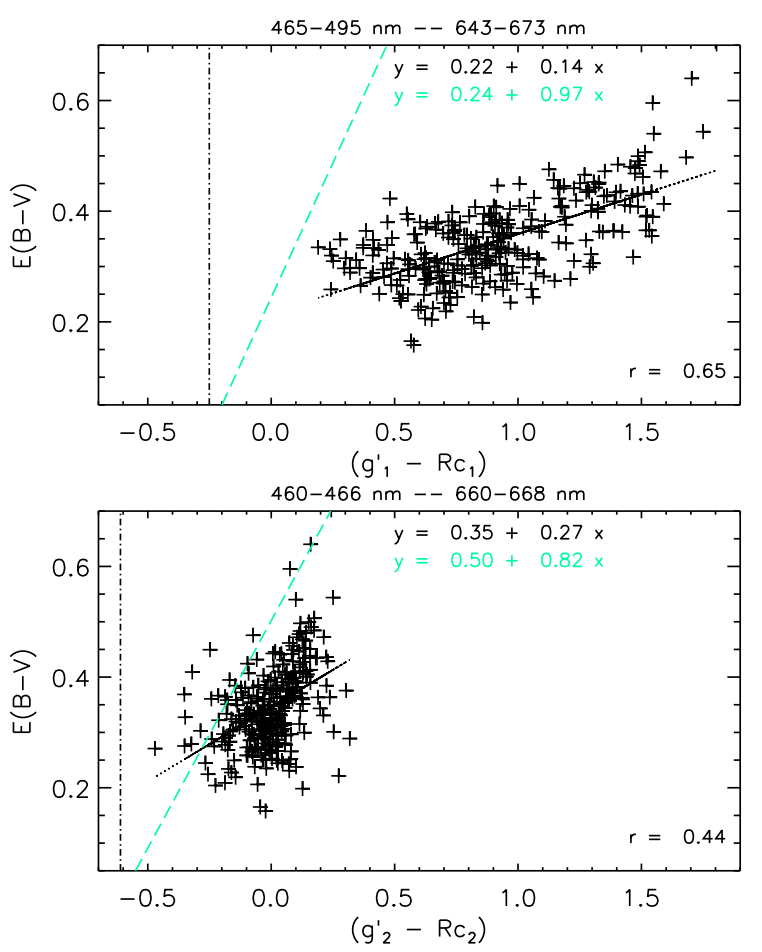

Fig. 4. Relation between the derived reddening and a colour similar to $g^{\prime}-R_{\mathrm{c}}$. The vertical line represent the $g^{\prime}-R_{\mathrm{c}}$ colour expected for a Im type galaxy without any extinction while the green dashed line corresponds to the expected relation if gas and stars were suffering the same amount of extinction (see text for details). The 1-degree polynomial fit to all the data appear as a continuous line. The corresponding fit is shown in the upper left corner while the Pearson's coefficient of correlation is indicated in the lower right corner. The simulated $g^{\prime}-R_{\mathrm{c}}$ colours have been done by integrating the flux in the spectral ranges of 4653-4953 $\AA\left(g_{1}^{\prime}\right)$ and 6431-6731 $\AA\left(R_{\mathrm{c} 1}\right)$ for the upper panel and $4600-4660 \AA\left(g_{2}^{\prime}\right)$ and $6600-6680 \AA\left(R_{\mathrm{c} 2}\right)$ for the lower one.

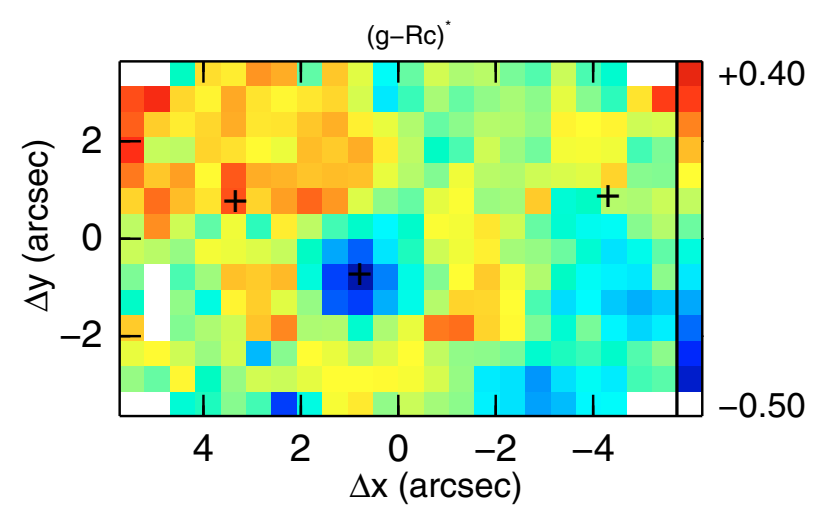

Fig. 5. Line free colour map. The simulated filters have been defined as explained in Fig. 4. The position of the three main peaks of continuum emission are shown for reference.

a factor 0.33 lower than the one for the ionized gas. This is similar to what Calzetti et al. (1997) found using HST images who estimated that the extinction suffered by the stars is a factor 0.5 lower than for the ionized gas and can be explained if the dust has a larger covering factor for the ionized gas than for the stars (Calzetti et al. 1994).

In general, our $E(B-V)$ measurements agree with previous ones using the same emission lines in specific areas (e.g. González-Riestra et al. 1987; López-Sánchez et al. 2007) or with poorer spatial resolution (Walsh \& Roy 1989). However there 
are discrepancies when comparing with the estimation of the extinction at other wavelengths. In particular, the peak of extinction ( $A_{V}=2.1 \mathrm{mag}$, according to the Balmer line ratio) is offset by $\sim 0 . ' 5$ from the peak of continuum emission. Alonso-Herrero et al. (2004) showed how in the central area of NGC 5253 there are two massive star clusters, $\mathrm{C} 1$ and $\mathrm{C} 2$. While $\mathrm{C} 1$ is the dominant source in the optical, coincident with our peak in the continuum map, the more massive and extinguished $\mathrm{C} 2$ is the dominant source in the infrared. The contours for the NICMOS F160W image in Fig. 3 show the good correspondence between our maximum of extinction and $\mathrm{C} 2$. Measurements in the near and mid-infrared suggest extinctions of $A_{V} \sim 17 \mathrm{mag}$ for this cluster (Turner et al. 2003; Alonso-Herrero et al. 2004; Martín-Hernández et al. 2005). The discrepancy between these two values indicates that a foreground screen model is not the appropriate one to explain the distribution of the dust in the giant $\mathrm{H}$ II region.

\subsection{Electron density distribution}

Electron density $\left(N_{\mathrm{e}}\right)$ can be determined from the [S II] $\lambda 6717 /$ [S II] $\lambda 6731$ and [Ar IV] $\lambda 4711 /[$ Ar IV] $\lambda 4740$ line ratios; values of 1.25 and 1.00 respectively were measured in a spectrum made from the sum of all our spaxels. Hereafter, we will refer to this spectrum as the integrated spectrum. Electron densities were determined assuming an electron temperature of $11650 \mathrm{~K}$, the average of the values given in López-Sánchez et al. (2007), and using the task temden, based on the fivel program (Shaw \& Dufour 1995) included in the IRAF package nebular. Derived values of $N_{\mathrm{e}}$ for the two line ratios were $180 \mathrm{~cm}^{-3}$ and $4520 \mathrm{~cm}^{-3}$, respectively. Differences between the electron densities derived from the argon and sulphur lines are usually found in ionized gaseous nebulae (see Wang et al. 2004) and are understood in terms of the ionization structure of the nebulae under study: [Ar IV] lines normally come from inner regions of higher ionization degree than [S II] lines. Typically, for giant Galactic and extragalactic $\mathrm{H}$ II regions, derived $N_{\mathrm{e}}$ from these two line ratios differ in a factor of $\sim 5$ (e.g. Esteban et al. 2002; Tsamis et al. 2003) which is much lower than what we find for the integrated spectrum of NGC 5253 ( 25). However, when only the giant $\mathrm{H}$ II region is taken into account (i.e. the area of $\sim 90$ spaxels where the argon lines are detected) the difference between the densities derived from the argon and the sulphur lines $(\sim 10$, see typical values for the densities below) is more similar to those found for other H II regions.

Maps for both ratios are shown in Fig. 6. According to the sulphur line ratio - detected over the whole field - densities range from very low values, of the order of the low density limit, in a region of about $5^{\prime \prime} \times 5^{\prime \prime}$ in the upper right corner of the field just above the Complex \#3, to $790 \mathrm{~cm}^{-3}$ at the peak of the emission in the cluster associated with the HII region, with a mean (median) over the field of view of $\sim 130$ (90) $\mathrm{cm}^{-3}$. The rest of the H II regions still present high densities (of about $400 \mathrm{~cm}^{-3}$ as a whole, $480 \mathrm{~cm}^{-3}$ in the $\mathrm{H} \mathrm{II-2}$, (i.e. the upper area of the giant H II region, Kobulnicky et al. 1997). This agrees well with the value estimated from long-slit measurements (López-Sánchez et al. 2007). The tail and the region associated with the cluster UV-1 present intermediate values (of about $200 \mathrm{~cm}^{-3}$ ).

The argon line ratio is used to sample the densest regions. The map for this ratio was somewhat noisier and allowed an estimation of the electron density only in the giant $\mathrm{H}$ II region. The densities derived from this line ratio are comparatively higher, with a mean (median) of $3400(3150) \mathrm{cm}^{-3}$. As it happened in the case of the extinction, the peak of electron density according
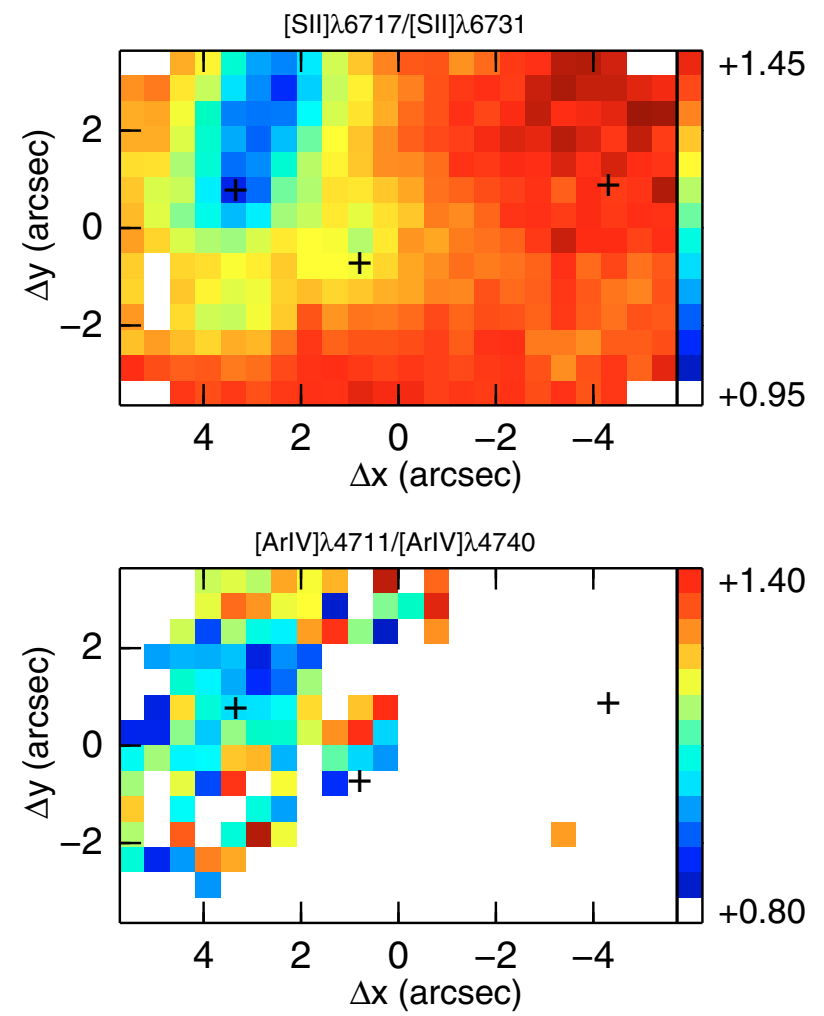

Fig. 6. Maps for the line ratios sensitive to the electron density. The position of the three main peaks of continuum emission are shown as crosses for reference. The displayed ranges in the line ratios imply electron densities of $<10-790 \mathrm{~cm}^{-3}$ and $190-8750 \mathrm{~cm}^{-3}$ for the sulphur and argon line ratio, respectively.

to this line ratio is offset by $\sim 0.7-11^{\prime \prime} 1$ towards the north-west with respect of the peak of continuum emission at Complex \#1.

We created higher $\mathrm{S} / \mathrm{N}$ ratio spectra by co-addition of $3 \times 3$ spaxel apertures associated with certain characteristic regions (i.e. the clusters at the core of the systems, $\mathrm{C} 1+\mathrm{C} 2$, and the regions H II-2, HII-1 and UV-1 of Kobulnicky et al. 1997). The largest values are measured around the core (i.e. $\mathrm{C} 1+\mathrm{C} 2)$ where the [Ar IV] electron density can be as high as $6200 \mathrm{~cm}^{-3}$. As we move further away from this region, the measured electron density becomes lower. Thus, H II-2 presents similar, although slightly lower, densities $\left(\sim 6100 \mathrm{~cm}^{-3}\right)$, followed by H II-1 with $\sim 4200 \mathrm{~cm}^{-3}$ and UV1 with $\sim 3300 \mathrm{~cm}^{-3}$. These values agree, within the errors, with those reported in López-Sánchez et al. (2007) for similar apertures.

An interesting point arises when the different density values derived for the integrated spectrum and for each individual spaxel/aperture are compared $\left(180 \mathrm{~cm}^{-3}\right.$, and up to $790 \mathrm{~cm}^{-3}$, respectively when using the sulphur line ratio). The covered f.o.v. $(\sim 210 \mathrm{pc} \times 135 \mathrm{pc})$ is comparable to the linear scales that one can resolve from the ground at distances of $\sim 40 \mathrm{Mpc}$ (or $z \sim$ 0.01). Such a comparison illustrates how aperture effects can cause important underestimation of the electron density in the $\mathrm{H}$ II regions in starbursts at such distances, or further away.

\subsection{Ionization structure, excitation sources and nitrogen enhancement}

The ionization structure of the interstellar medium can be studied by means of diagnostic diagrams. Different areas of a given diagram are explained by different ionization mechanisms. In the 

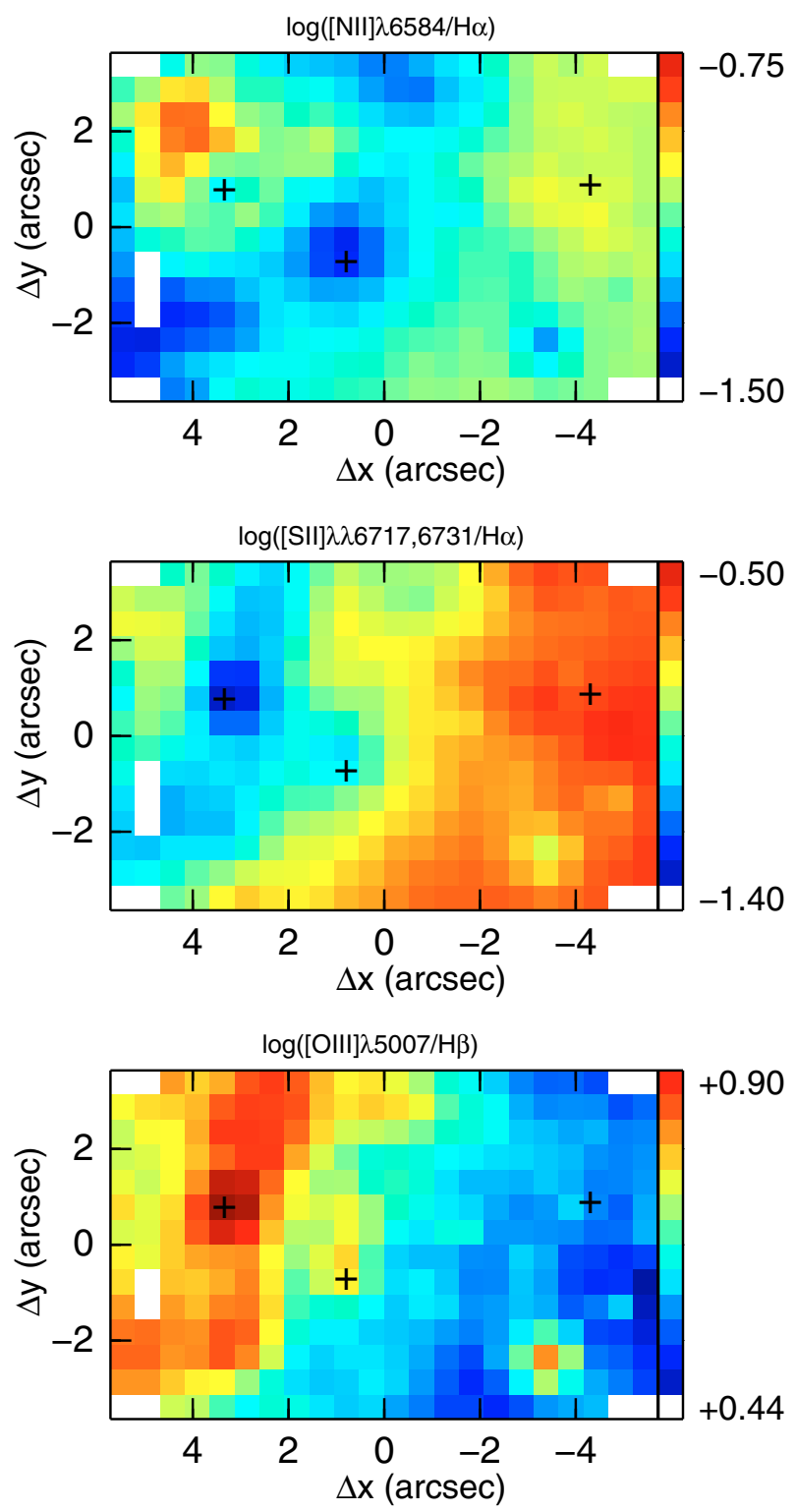

Fig. 7. Emission line ratio maps. Up: $\log ([\mathrm{N} \mathrm{II}] \lambda 6584 / \mathrm{H} \alpha)$. Middle: $\log ([\mathrm{S} \mathrm{II}] \lambda \lambda 6717,6731 / \mathrm{H} \alpha)$. Bottom: $\log ([\mathrm{O} \mathrm{III}] \lambda 5007 / \mathrm{H} \beta)$. The position of the three main peaks of continuum emission are shown for reference.

optical spectral range, the most widely used are probably those proposed by Baldwin et al. (1981) and later reviewed by Veilleux \& Osterbrock (1987), the so-called BPT diagrams. In Fig. 7 the maps for the three available line ratios involved in these diagrams - namely [N II] $\lambda 6584 / \mathrm{H} \alpha,[\mathrm{S}$ II] $\lambda \lambda 6717,6731 / \mathrm{H} \alpha$, [O III $] \lambda 5007 / \mathrm{H} \beta-$ are shown on a logarithmic scale. This figure shows that the ionization structure in the central region of this galaxy is complex. Not only do the line ratios not show a uniform distribution, but the structure changes depending on the particular line ratio.

Both the $[\mathrm{O}$ III $] \lambda 5007 / \mathrm{H} \beta$ and the $[\mathrm{S} \mathrm{II}] \lambda \lambda 6717,6731 / \mathrm{H} \alpha$ line ratios display a gradient away from the peak of emission at Complex \#1 and with a structure that follows that of the ionized gas. Thus the $[\mathrm{S} \mathrm{II}] \lambda \lambda 6717,6731 / \mathrm{H} \alpha([\mathrm{O}$ III $] \lambda 5007 / \mathrm{H} \beta)$ ratio is smallest (largest) at Complex \#1, presents somewhat intermediate values in the two tongue-shaped extensions and the Complex \#2 and is relatively high (low) in the rest of the
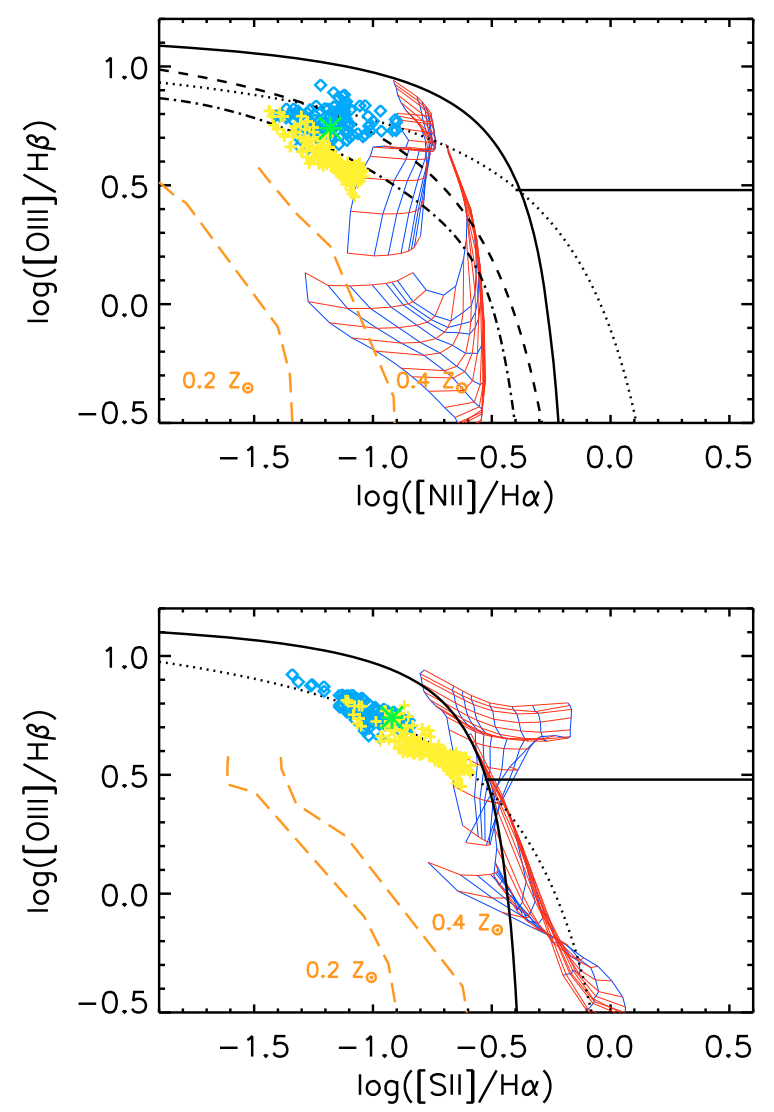

Fig. 8. Position of the individual spaxels in NGC 5253 in the diagnostic diagrams proposed by Veilleux \& Osterbrock (1987). Data points above and below the 3- $\sigma$ level of the first-degree polynomial fit in the $[\mathrm{N} \mathrm{II}] \lambda 6584 / \mathrm{H} \alpha$ vs. [S II] $\lambda \lambda 6717,6731 / \mathrm{H} \alpha$ diagram (see text for details) have been represented with blue diamonds and yellow crosses respectively. The solid curves show the empirical borders found by Veilleux \& Osterbrock between ionization caused by different mechanisms, while the dotted lines show the theoretical borders proposed by Kewley et al. (2001) to delimit the area where the line ratios can be explained by star formation. Black dashed and dot-dashed lines show the revised borders by Kauffmann et al. (2003) and Stasińska et al. (2006), respectively. Solid horizontal lines show the border between classical Seyfert galaxies (above the line) and LINERs (below the line), at $[\mathrm{O} \mathrm{III}] \lambda 5007 / \mathrm{H} \beta=3$. Long-dashed lines in orange represent the predictions from models of photo-ionization by stars presented in Dopita et al. (2006) for $R=0$ and ages of 2-6 Myr. The grids represent the predictions for the shock models presented in Allen et al. (2008), with and without precursor, assuming the abundances of the LMC and $N_{\mathrm{e}}=1 \mathrm{~cm}^{-3}$. Lines of constant magnetic parameter (blue) cover a range of $B=0.0001-10 \mu \mathrm{G}$, while lines of constant shock velocity (red) cover a range of $v_{\mathrm{s}}=150-500 \mathrm{~km} \mathrm{~s}^{-1}$. Green asterisks mark the derived values for the integrated spectrum of the whole IFU field.

field, with a secondary minimum (maximum) at $\sim\left[-33^{\prime \prime} 5,-2\right.$. '5], the position of a secondary peak in the $\mathrm{H} \alpha$ emission. This is coincident with the structure presented in (Calzetti et al. 2004).

The $[\mathrm{N} \mathrm{II}] \lambda 6584 / \mathrm{H} \alpha$ line ratio, however, display a different structure. While in the right half of the FLAMES field of view, the behavior is quite similar to the one observed for the $[\mathrm{S}$ II] $\lambda \lambda 6717,6731 / \mathrm{H} \alpha$ ratio (i.e. values relatively high, local minimum at $\left.\sim\left[-33^{\prime \prime} 5,-2{ }^{\prime \prime} 5\right]\right)$, the left half, dominated by the giant H II region, displays a completely different pattern. The lowest values are associated with Complex \#2 and the southern extension, rather than Complex \#1, and the [N II] $66584 / \mathrm{H} \alpha$ line ratios are highest at $\sim\left[4{ }^{\prime \prime} 0,2\right.$ '. $\left.^{\prime} 0\right]$. 
In Fig. 8 we show the position of each spaxel of the FLAMES field of view, as well as for the integrated spectrum, after co-adding all the spaxels in the BPT diagnostic diagrams together with the borders that separate H II region-like ionization from ionization by other mechanisms according to several authors (Veilleux \& Osterbrock 1987; Kewley et al. 2001; Kauffmann et al. 2003; Stasińska et al. 2006). We also show the predictions for models of photo-ionization caused by stars (Dopita et al. 2006) that take into account the effect of the stellar winds on the dynamical evolution of the region. In these models, the ionization parameter is replaced by a new variable $R$ that depends on the mass of the ionizing cluster and the pressure of the interstellar medium $\left(R=\left(M_{\mathrm{Cl}} / M_{\odot}\right) /\left(P_{o} / k\right)\right.$, with $P_{o} / k$ measured in $\left.\mathrm{cm}^{3} \mathrm{~K}\right)$. Also, the predictions for shocks models for a LMC metallicity are included. Given the relatively low metallicity of NGC 5253, these are the most appropriate ones. They were calculated assuming a $N_{\mathrm{e}}=1 \mathrm{~cm}^{-3}$ and cover an ample range of magnetic parameters, $B$, and shock velocities, $v_{\mathrm{s}}$, (see Allen et al. 2008, for details).

As demonstrated for the electron density, these diagrams illustrate very clearly how resolution effects can influence the measured line ratios. Values derived for individual spaxels cover a range of $\sim 0.5,1.0$ and $0.5 \mathrm{dex}$ for the $[\mathrm{N} \mathrm{II}] \lambda 6584 / \mathrm{H} \alpha$, $[\mathrm{S} \mathrm{II}] \lambda \lambda 6717,6731 / \mathrm{H} \alpha$, and $[\mathrm{O} \mathrm{III}] \lambda 5007 / \mathrm{H} \beta$ line ratios respectively, with mean values similar to the integrated values $(-1.15,0.92$, and 0.74$)$. This is particularly relevant when interpreting the ionization mechanisms in galaxies at larger distances where the spectrum can sample a region with a range in ionization properties. This loss of spatial resolution thus "smears" the determination of the ionization mechanism by a set of line ratios. Even if this given set of line ratios is typical of photoionization caused by stars, it is not possible to exclude some contribution due to other mechanisms at scales unresolved by the particular observations.

Regarding the individual measurements, although all line ratios are within the typical values expected for an $\mathrm{H}$ II region-like ionization, two differences between these diagrams arise. The first one is that the diagram involving the $[\mathrm{S} \mathrm{II}] \lambda \lambda 6717,6731 / \mathrm{H} \alpha$ line ratio indicates a somewhat higher ionization degree than the one involving the $[\mathrm{N} \mathrm{II}] \lambda 6584 / \mathrm{H} \alpha$ line ratio. That is: values for the diagram involving the $[\mathrm{S}$ II] $] \lambda \lambda 6717,6731 / \mathrm{H} \alpha$ line ratio are at the limit of what can be explained by pure photo-ionization in an H II region according to the Kewley et al. (2001) theoretical borders. On the contrary, most of the data points in the diagram involving the $[\mathrm{N}$ II $] \lambda 6584 / \mathrm{H} \alpha$ are clearly in the area associated to photoionization caused by stars. A comparison with the predictions of the models for metallicities similar to the one of NGC 5253 shows how the measured line ratios present intermediate values between those predicted by ionization caused by shocks and those by pure stellar photoionization. That this is exactly what one would expect if shocks caused by the mechanical input from stellar winds or supernovae within the starburst were contributing to the observed spectra. Also, this comparison supports previous studies that show how models of photoionization caused by stars underpredict the $[\mathrm{O} \mathrm{III}] \lambda 5007 / \mathrm{H} \beta$ line ratios, specially in the low-metallicity cases (Brinchmann et al. 2008; Dopita et al. 2006).

The second diference is the distribution of the data points in these diagrams. While the data points in the [S II] $\lambda \lambda 6717,6731 / \mathrm{H} \alpha$ vs. [O III] $] 25007 / \mathrm{H} \beta$ diagram form a sequence, data points in the $[\mathrm{N} \mathrm{II}] \lambda 6584 / \mathrm{H} \alpha$ vs. [O III] $\lambda 5007 / \mathrm{H} \beta$ diagram are distributed in two groups: a sequence similar to the one in the $[\mathrm{S} \mathrm{II}] \lambda \lambda 6717,6731 / \mathrm{H} \alpha$ vs. [O III $] \lambda 5007 / \mathrm{H} \beta$

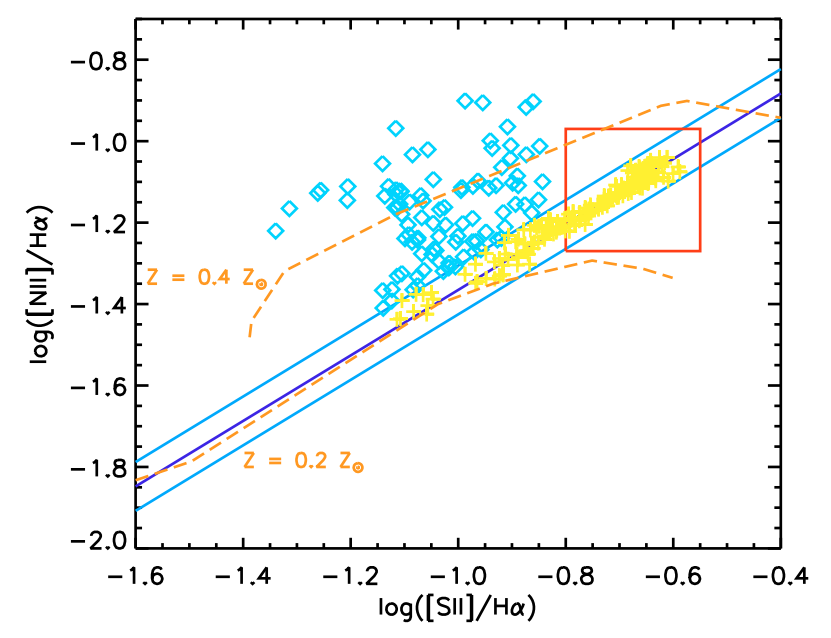

Fig. 9. [S II] $\lambda \lambda 6717,6731 / \mathrm{H} \alpha$ vs. [N II] $] 6584 / \mathrm{H} \alpha$ diagram. The red box includes the data points utilized to determine the first-degree polynomial fit (dark blue continuous line) between the [S II] $\lambda \lambda 6717,6731 / \mathrm{H} \alpha$ and $[\mathrm{N}$ II $] \lambda 6584 / \mathrm{H} \alpha$ line ratios. Data points above and below the 3- $\sigma$ level (light blue continuous line) have been represented with blue diamonds and yellow crosses respectively. The expected line ratios for $\mathrm{H}$ II regions for $Z=0.2 Z_{\odot}$ and $Z=0.4 Z_{\odot}$ with ages in the range of 2-6 Myr and $R=0$ according to the models presented in Dopita et al. (2006) are over-plotted with dashed oranges lines for comparison.

diagram and a cloud of data points above that sequence with larger [N II] $\lambda 6584 / \mathrm{H} \alpha$. This result can be interpreted either by local variations in the relative abundances or by changes in the ionization parameter. Here we will explore the first option, which is the most accepted explanation (e.g. López-Sánchez et al. 2007, and references therein) and is supported by the relatively constant ionization parameter found in specific areas via long-slit $(\log (U) \sim-3$, Kobulnicky et al. 1997). Long-slit measurements in specific areas of this field have shown out how this galaxy present some regions with an over-abundance of nitrogen (e.g. Walsh \& Roy 1989; Kobulnicky et al. 1997). For our measured line ratios and using expression (22) in Pérez-Montero $\&$ Contini (2009), we measure a range in $\log (\mathrm{N} / \mathrm{O})$ of -0.70 to -1.46 . Here we will assume that this over-abundance is the cause of our excess in the $[\mathrm{N} \mathrm{II}] \lambda 6584 / \mathrm{H} \alpha$ line ratio and will use this excess to precisely delimit the area presenting this overabundance. To this aim, we placed the information of each of the spaxels in the $[\mathrm{S} \mathrm{II}] \lambda \lambda 6717,6731 / \mathrm{H} \alpha$ vs. [N II] $] \lambda 6584 / \mathrm{H} \alpha$ diagram, which better separates the two different groups described above. This is presented in Fig. 9. We have assumed that in the so-called un-polluted areas, the [N II] $66584 / \mathrm{H} \alpha$ and [S II] $\lambda \lambda 6717,6731 / \mathrm{H} \alpha$ follow a linear relation. This is a reasonable assumption since the $[\mathrm{N}$ II] $] \lambda 6584 /[\mathrm{S}$ II $] \lambda \lambda 6717,6731$ line ratio has a low dependence with the abundance and the properties of the ionizing radiation field (Kewley \& Dopita 2002). This standard relation was determined by fitting a first-degree polynomial to the data points with [S II] $\lambda \lambda 6717,6731 / \mathrm{H} \alpha>-0.8$ (indicated in Fig. 9 with a red box). Those spaxels whose $[\mathrm{N} \mathrm{II}] \lambda 6584 / \mathrm{H} \alpha$ line ratio was in excess of more than $3-\sigma$ from the relation determined by this fit, have been identified as having an [N II] $\lambda 6584 / \mathrm{H} \alpha$ excess, and are identified by diamonds in Fig. 9. As can be seen from this figure, there are a number of spaxels where this excess is much above the standard relation.

The data points thus identified with [N II] $\lambda 6584 / \mathrm{H} \alpha$ excess are shown as a map in Fig. 10 where the location and magnitude of the $[\mathrm{N} \mathrm{II}] \lambda 6584 / \mathrm{H} \alpha$ excess is indicated by white circles, 


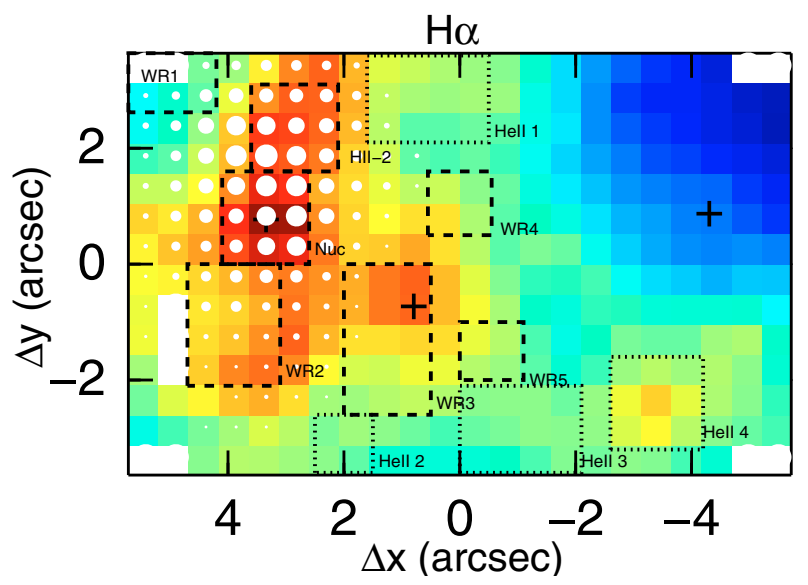

Fig. 10. Spaxels with $[\mathrm{N}$ II] $\lambda 6584 / \mathrm{H} \alpha$ line ratio above the $3-\sigma$ level of the fit presented in Fig. 9 on top of our $\mathrm{H} \alpha$ emission line map. The size of the white circles is proportional to the excess in the $[\mathrm{N}$ II $] \lambda 6584 / \mathrm{H} \alpha$ line ratio. Dashed and dotted lines delimit the apertures utilized to extract the spectra with Wolf-Rayet and nebular He II features displayed in Figs. 11 and 12 (see Sects. 3.5 and 3.6). The position of the three peaks of continuum emission in the map are shown by crosses for reference.

whose size is proportional to the size of the $[\mathrm{N} \mathrm{II}] \lambda 6584 / \mathrm{H} \alpha$ excess. This figure can be interpreted as a snapshot in the pollution process of the interstellar medium by the SSCs in the central area of NGC 5253. The pollution is affecting almost the whole giant $\mathrm{H}$ II region. The largest values are found at $\sim 1$ ". 5 towards the north-west of the peak at Complex \#1. Then, the quantity of extra nitrogen decreases outwards following the two tongueshaped extensions towards the north-west and south-east. This is consistent with the HST observations of Kobulnicky et al. (1997) who found nitrogen enrichment in their H II- 1 and $\mathrm{H} \mathrm{II-2}$, while the N/O ratio in UV-1 (see Table 3) was typical for metal-poor galaxies.

\subsection{Wolf-Rayet features}

Wolf-Rayet (W-R) stars are very bright objects with strong broad emission lines in their spectra. They are classified as WN (those with strong lines of helium and nitrogen) and WC (those with strong lines of helium, carbon and oxygen) and are understood as the result of the evolution of massive $\mathrm{O}$ stars. As they evolve, they loose a significant amount of their mass via stellar winds showing the products of the CNO-burning first - identified as WN stars - and the He-burning afterwards - as WC stars (Conti 1976). The presence of W-R stars can be recognized via the W-R bumps around $\lambda 4650 \AA$ (i.e. the blue bump, characteristic of WN stars) and $\lambda 5808 \AA$ (i.e. the red bump, characteristic of WC stars, but not covered by the present data).

Schaerer et al. $(1997,1999)$ carried out a thorough search and characterization of the W-R population in NGC 5253. They detected W-R features (both WN and WC) at the peak of emission in the optical (our Complex \#1) and the ultraviolet (our Complex \#2). Due to the spatial coincidence of these detections and the N-enriched regions found by Walsh \& Roy (1989) and Kobulnicky et al. (1997) - at least in the case of the nucleus they suggested that these W-R stars could be the cause of this enhancement. This is supported observationally by similar findings in other W-R galaxies. For example, a recent survey using Sloan data of W-R galaxies in the low-redshift Universe has shown that galaxies belonging to this group present an elevated N/O ratio, in comparison with similar non-W-R galaxies (Brinchmann et al. 2008). Other suggested possibilities to cause the enrichment in nitrogen include planetary nebulae, $\mathrm{O}$ star winds, He-deficient W-R star winds, and luminous blue variables (Kobulnicky et al. 1997).

Here, we characterize the W-R population in NGC 5253 and explore the hypothesis of W-R stars as the cause of the nitrogen enhancement by using the $2 \mathrm{D}$ spectral information provided by the present data. In the previous section we have delimited very precisely the area that presents nitrogen enhancement. In a same manner, it is possible to look for and localize the areas that present W-R emission. Note that due to the continuous sampling of the present data this can be done in a completely unbiased way.

We visually inspected each spectrum looking for the more prominent W-R features in the blue bump (i.e. N III $\lambda 4640$ and He II 14686). The areas where these features have been found are marked in Fig. 10 with dashed lines. The co-added and extracted spectra of each individual area appear in Fig. 11 together with a reference spectrum, free of $\mathrm{W}-\mathrm{R}$ features, made by coadding 20 spaxels in the upper right corner of the FLAMES field of view.

We confirm the detection of W-R features associated with the nucleus and the brightest cluster in the ultraviolet, UV-1 (our W-R 3). In the same manner, we also detect a broad He II line associated with the north-west and south-east extensions (i.e. H II-2 and W-R 2, respectively). In addition, there are three more areas which present W-R features, called W-R 1 , W-R 4 and W-R 5, relatively far $(\sim 58-83 \mathrm{pc})$ from the main area of activity. Interestingly, two of these regions (W-R 4 and W-R 5) present a narrow nebular He II on top of the broad W-R feature.

The short phase of W-R stars during star evolution makes their detection a very precise method for estimating the age of a given stellar population. According to Leitherer et al. (1999), typically an instantaneous starburst shows these features at ages of 3-6 Myr for metallicites of $Z=0.004-0.008$, similar to the one in NGC 5253. Thus, very young stellar clusters must be associated with the areas that display these W-R features. We compared the positions of our detections with the catalogue of clusters given by Harris et al. (2004) and compiled in Table 4. All our regions, except W-R 5, are associated with one (or several) young (i.e. <5 Myr) star cluster(s). Regarding W-R 5, Harris et al. (2004) do not report any cluster associated with that area.

Cluster ages in Harris et al. (2004) were estimated using both broadband photometry and the $\mathrm{H} \alpha$ equivalent width. We estimated the ages by means of two indicators: the ratio between the number of $\mathrm{W}-\mathrm{R}$ and $\mathrm{O}$ stars; and the $\mathrm{H} \beta$ equivalent width. The ratio between the number of $\mathrm{W}-\mathrm{R}$ and $\mathrm{O}$ stars was estimated from $F(\mathrm{bb}) / F(\mathrm{H} \beta)$, where $F(\mathrm{bb})$ and $F(\mathrm{H} \beta)$ are the flux in the blue bump (measured with splot) and in $\mathrm{H} \beta$ respectively and using the relation proposed by Schlegel et al. (1998). Uncertainties are large, mainly due to the difficulty to define the continuum and to avoid the contamination of the nebular emission lines when measuring $F(\mathrm{bb})$ but indicates a range in $\log (\mathrm{WR} /(\mathrm{WR}+\mathrm{O}))$ of $\sim-2.0$ to -1.2 (see Table 4 , Col. 4). There is a higher proportion of W-R stars in W-R 5, W-R 1 and W-R 4 and somewhat lower in those areas associated with the giant $\mathrm{H}$ II region. The $\mathrm{H} \beta$ equivalent widths are extremely high, consistent again with the expected youth of the stellar population. Predicted ages from these two age tracers are reported in Table 4, Cols. 5 and 7. They were estimated by using STARBURST99 (Leitherer et al. 1999), assuming an instantaneous burst of $Z=0.008$, an upper mass limit of $M_{\text {up }}=100 M_{\odot}$ 

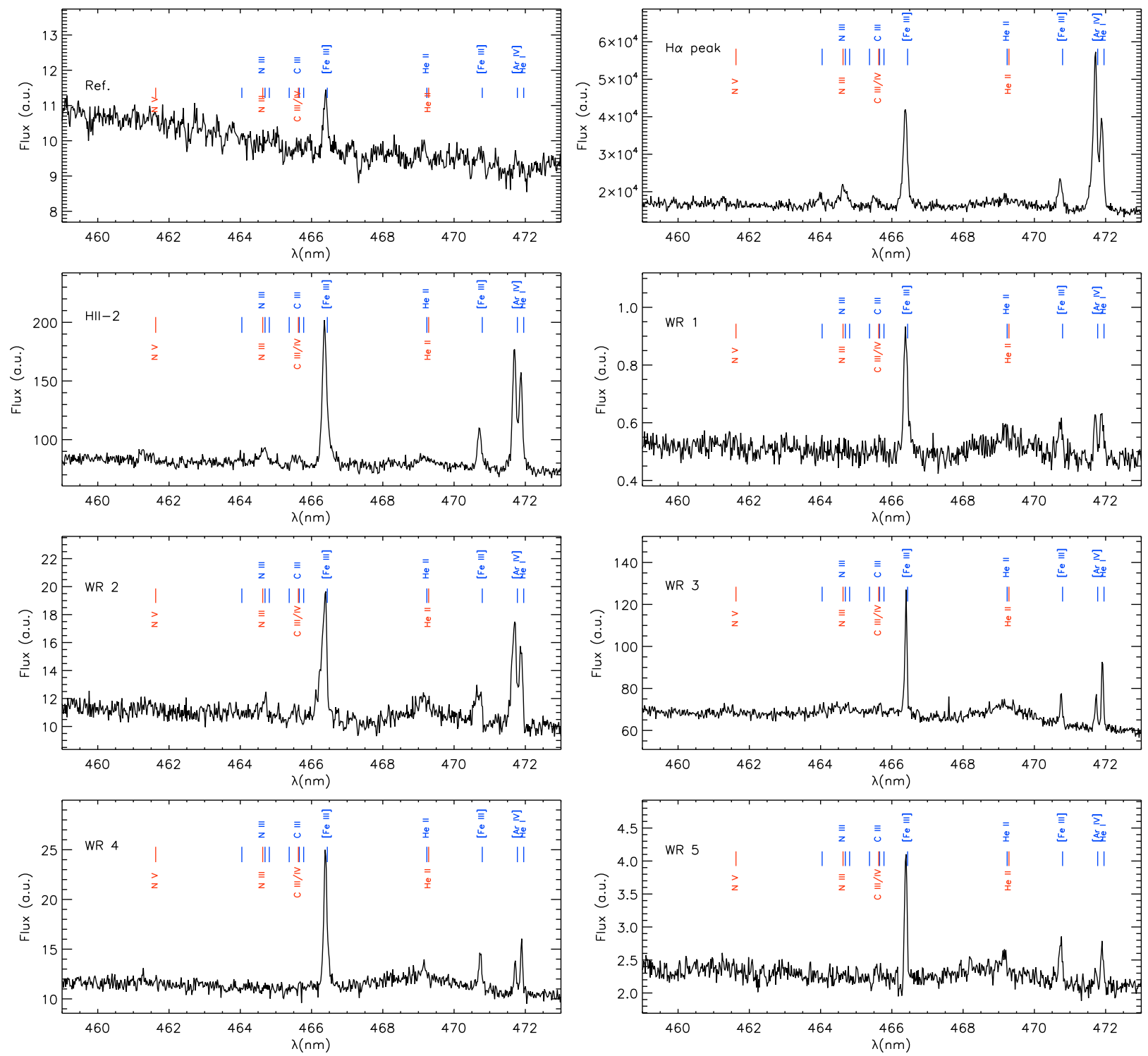

Fig. 11. Spectra showing Wolf-Rayet features. The first spectrum has been extracted from a low surface brightness area in the upper right corner of the FLAMES field of view and is presented here as reference. The positions of the nebular emission lines have been indicated with blue ticks and labels, while those corresponding to Wolf-Rayet features appear in red. The position and extent of the regions are indicated in Fig. 10.

Table 4. Ages of the clusters associated with the W-R regions according to different indicators.

\begin{tabular}{ccccccc}
\hline \hline Region & $\begin{array}{c}\text { Harris et al. (2004) } \\
\text { Clusters } \\
\text { Number }\end{array}$ & $\begin{array}{c}\text { Age } \\
(\mathrm{Myr})\end{array}$ & $\log (\mathrm{WR} /(\mathrm{WR}+\mathrm{O}))$ & $\begin{array}{c}\text { This work } \\
\mathrm{Age}_{\mathrm{WR} / \mathrm{O}} \\
(\mathrm{Myr})\end{array}$ & $\begin{array}{c}E W(\mathrm{H} \beta) \\
(\AA)\end{array}$ & $\begin{array}{c}\mathrm{Age}_{\mathrm{H} \beta} \\
(\mathrm{Myr})\end{array}$ \\
\hline Nucleus & 1 & 3 & -1.99 & 2.9 & 245 & 2.7 \\
H II-2 & 20 & 3 & -1.93 & 2.9 & 320 & 2.4 \\
W-R 1 & 13 & 4 & -1.34 & $3.2 / 4.8$ & 131 & 3.4 \\
W-R 2 & 23 & 5 & -1.64 & $3.0 / 5.5$ & 180 & 3.2 \\
W-R 3 & $4,24,25$ & $1-5$ & -1.55 & $3.0 / 5.3$ & 128 & 3.4 \\
W-R 4 & 6,21 & 4 & -1.35 & $3.2 / 4.8$ & 131 & 3.4 \\
W-R 5 & $\ldots$ & $\ldots$ & -1.17 & 3.4 & 86 & 4.7 \\
\hline
\end{tabular}

and a Salpeter-type Initial Mass Function. The two age tracers give consistent age predictions and in agreement with those reported in Harris et al. (2004).

The distribution of the W-R features, in an area of about $100 \mathrm{pc} \times 100 \mathrm{pc}$, much larger than the one polluted with nitrogen, suggests that all the detected W-R stars are not, in general, the cause of this pollution. Since the N-enrichment appears to be associated with the pair of clusters in the core and, given the position of maximum, most probably with the obscured SSC C2, the best W-R star candidates to be the cause of this enrichment are those corresponding to our Nuc aperture, and perhaps also the $\mathrm{H}$ II- 2 and W-R 2 regions. 

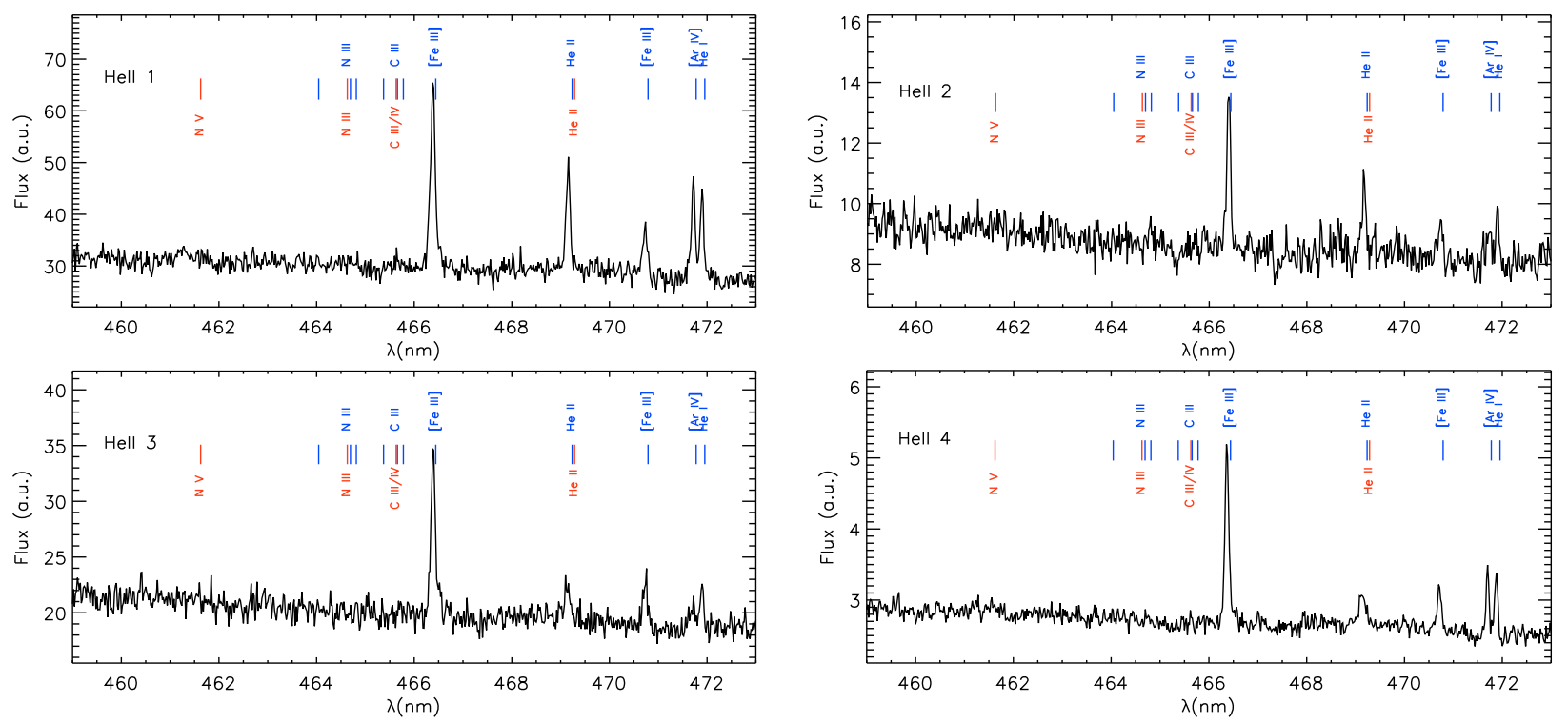

Fig. 12. Spectra showing nebular He II, but no W-R features. The positions of the nebular emission lines have been indicated with blue ticks and labels, while those corresponding to Wolf-Rayet features appear in red.

\subsection{Nebular He II and helium abundance}

The hypothesis that the W-R population is the cause of the nitrogen enrichment in NGC 5253 requires an enhancement of the helium abundance too (e.g. Schaerer 1996). This is nicely illustrated in Kobulnicky et al. (1997) where different linear relations between the nitrogen and helium abundances $(\mathrm{N} / \mathrm{H}$ and $\mathrm{He} / \mathrm{H})$ are presented according to different scenarios of nitrogen enrichment (W-Rs, PNe, etc.). The only scenario able to explain an extra quantity of nitrogen in the ISM without any extra helium counterpart would be the one where this nitrogen is caused during the late O-star wind phase.

As in previous sections, we can measure at each spaxel the total helium abundance and compare it with that for nitrogen. Since lines like [O II] $\lambda \lambda 3726,3728$ did not fall in the covered spectral range, we did not determine the absolute nitrogen abundance. Instead, we used the mean of the abundances determined by Kobulnicky et al. (1997) for their H II-1 and H II-2 $\left(\mathrm{N} / \mathrm{H} \sim 2.0 \times 10^{-5}\right)$ to estimate how much helium would be needed in the enriched areas, if the extra nitrogen were caused by $\mathrm{W}$-Rs (i.e. $\mathrm{He} / \mathrm{H} \sim 0.12$ ). For the non $\mathrm{N}$-enriched areas, we can use the measurement at UV-1 $\left(\mathrm{N} / \mathrm{H} \sim 0.7 \times 10^{-5}\right)$ which requires $\mathrm{He} / \mathrm{H} \sim 0.09$. Helium abundance can be determined as:

$\mathrm{He} / \mathrm{H}=\operatorname{icf} \times\left(\mathrm{He}^{+} / \mathrm{H}^{+}+\mathrm{He}^{++} / \mathrm{H}^{+}\right)$

where icf is a correction factor due to the presence of neutral helium. We assumed icf $\sim 1.0$, which is consistent with the predictions of photoionization models for our measured $[\mathrm{O}$ III $] \lambda 5007 / \mathrm{H} \beta$ line ratios (Holovatyy \& Melekh 2002). Since the He I 26678 was detected in every spaxel of the FLAMES field of view and with good $\mathrm{S} / \mathrm{N}$, for the purpose of this work, we determined $y^{+}=\mathrm{He}^{+} / \mathrm{H}^{+}$from the $\mathrm{He} \mathrm{I} \lambda 6678 / \mathrm{H} \alpha$ line ratio using the expression

$y^{+}=2.58 t^{0.25}(2.87 \mathrm{He} \mathrm{I} \lambda 6678 / \mathrm{H} \alpha)$

where $t$ is the electron temperature in units of $10^{4} \mathrm{~K}$ (Pagel et al. 1992). As in Sect. 3.3, we assumed $T_{\mathrm{e}}=11650 \mathrm{~K}$. Figure 13 shows the 2D structure of the $\mathrm{He} \mathrm{I} \lambda 6678 / \mathrm{H} \alpha$ line ratio. It is relatively uniform with the exception of some spaxels

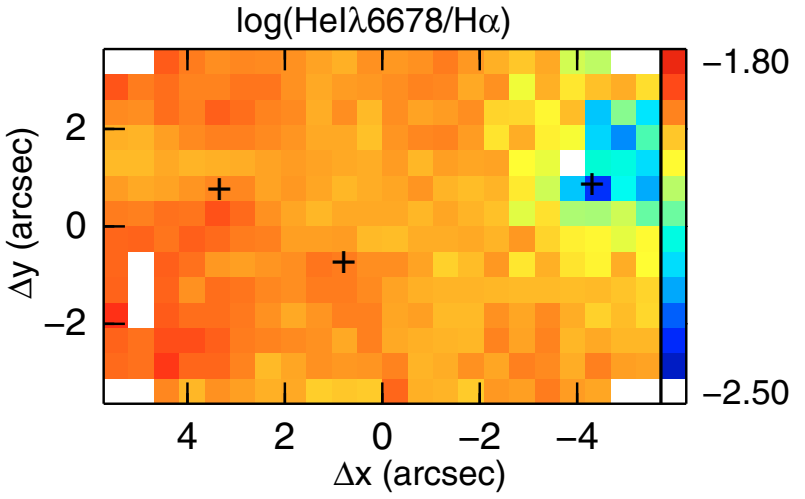

Fig. 13. $\log (\mathrm{He} \mathrm{I} \lambda 6678 / \mathrm{H} \alpha)$ line ratio map. The position of the three peaks in the continuum map are shown for reference.

in the upper right corner, close to Complex \#3. This area, relatively far from the main photo-ionization source and with low [O III] $] \lambda 5007 / \mathrm{H} \beta$ line ratio would be the only region where one can expect a substantial contribution of neutral helium.

Figure 14 presents the derived $\mathrm{He}^{+}$abundances vs. the $[\mathrm{O}$ III $] \lambda 5007 / \mathrm{H} \beta$ line ratio. With the exception of the data corresponding to the right upper corner of the FLAMES f.o.v., $\mathrm{He}^{+} / \mathrm{H}^{+}$range between 0.075 and 0.090 , being higher in the higher excitation zones (i.e. the giant $\mathrm{HII}$ region). These values are in agreement with previous measurements of $\mathrm{He}^{+} / \mathrm{H}^{+}$in specific areas (Pagel et al. 1992; Kobulnicky et al. 1997; Walsh \& Roy 1989). They are consistent with a scenario without extra $\mathrm{N}$-enrichment and still far, by a factor $\sim 1.3-1.7$, from the required $\sim 0.12$ total helium abundance in the W-R scenario, in particular in the areas enriched with nitrogen.

What about the $\mathrm{He}^{++} / \mathrm{H}^{+}$, whose abundance can be determined via the nebular He II $\lambda 4686$ emission line? This line turned out to be rather elusive. Campbell et al. (1986) mentioned a possible detection in their regions B and C. This result, however, has not been confirmed afterwards (see López-Sánchez et al. 2007, and references therein). As with the W-R features, we looked for the He II nebular line in each individual spectrum. Those that presented a spatial continuity were taken to define an area and 


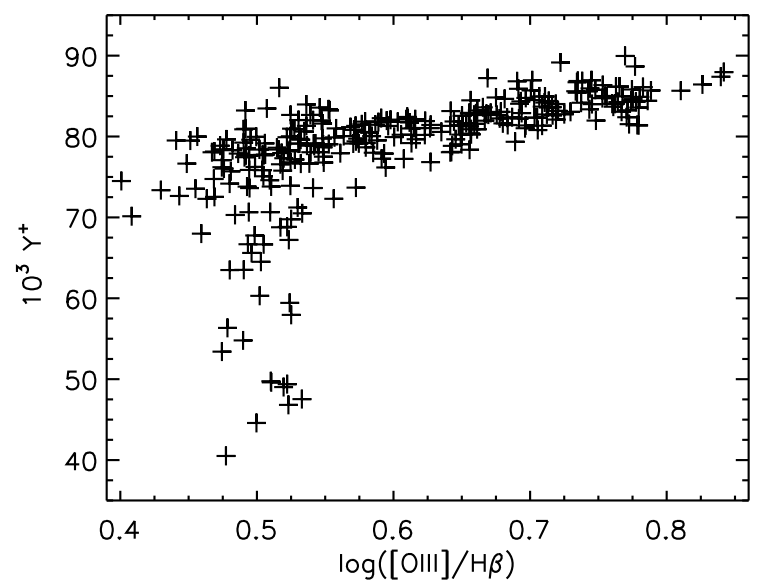

Fig. 14. $\mathrm{He}^{+}$abundance vs. [O III $] \lambda 5007 / \mathrm{H} \beta$ for each individual spaxel.

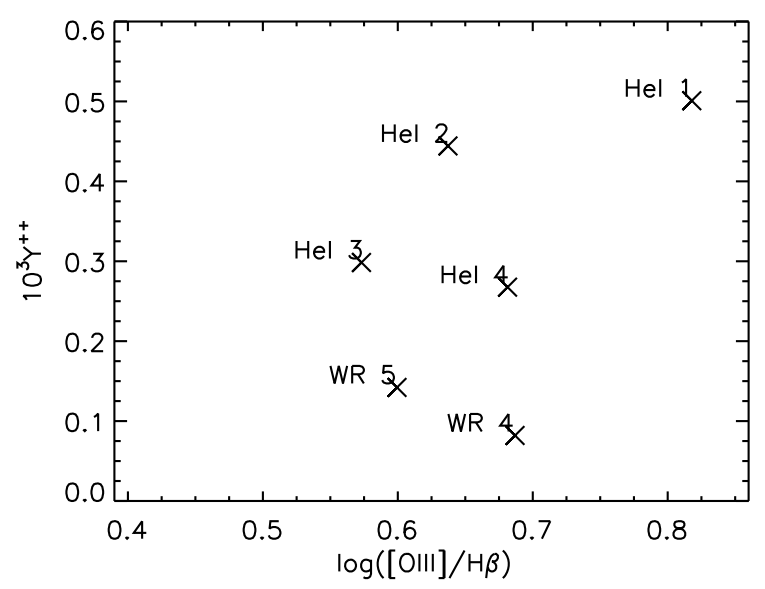

Fig. 15. $\mathrm{He}^{++}$abundance vs. $[\mathrm{O} \mathrm{III}] \lambda 5007 / \mathrm{H} \beta$ for each of the extracted spectra with He II $\lambda 4686$ detection (dotted regions in Fig. 10).

were co-added before extracting. The selected areas are marked in Fig. 10 with dotted lines. The co-added and extracted spectra of each individual region appear in Fig. 12. In addition to these regions, as mentioned in Sect. 3.5, nebular He II in the W-R 4 and W-R 5 has also been detected.

These detections are, in general, neither associated with the area of nitrogen enhancement nor with those presenting W-Rs features. This lack of coincidence seems difficult to reconcile with a scenario where this enhancement, and the existence of $\mathrm{He}^{++}$, share a common origin. Moreover, for the purpose of this work, we estimated the He II abundances in this areas using:

$y^{++}=0.084 t^{0.14}(\mathrm{He}$ ІІ $\lambda 4686 / \mathrm{H} \beta)$

from Pagel et al. (1992). Derived values for the individual regions are shown Fig. 15 as a function of the $[\mathrm{O} \mathrm{III]}] \lambda 5007 / \mathrm{H} \beta$ line ratio. They range between 0.0001 and 0.0005 . Although uncertainties are large, up to 0.0006 , due to the weakness of the He II $\lambda 4686$ emission line, these values are clearly very far from the values of $\sim 0.030-0.050$ required to bring the helium abundance up to $\sim 0.12$ in the W-R enrichment scenario. Given the depth and continuous mapping of the present data, we can clearly exclude the possibility of further detections of larger quantities of $\mathrm{He}^{++}$based on optical observations. Thus the present data support the scenario suggested by Kobulnicky et al. (1997) where the N-enrichment should arise during the late Ostar wind phase. In view of the extra-nitrogen distribution and the extinction map, the only place where these larger quantities of $\mathrm{He}^{++}$could be found (if they existed) is in Complex \#1. However, they should be highly extinguished and would required a search for emission lines at longer wavelengths as for example He II (7-10) at $21891 \AA$ (Hora et al. 1999).

Since the nebular He II is not associated with the area showing $\mathrm{N}$-enhancement, there is still the open question as to its origin. Garnett et al. (1991) explored the different mechanisms capable of producing this emission in extragalactic H II regions. The first suggestion is photoionization by fast shocks. However, we have seen in Sect. 3.4, that shocks do not appear to play a dominant role in the central parts of NGC 5253. Moreover, the measured $\log (\mathrm{He} \mathrm{II} \lambda 4686 / \mathrm{H} \beta)$ are $\sim-3.0$ to -2.1 , much lower than those predicted by shocks models with $N_{\mathrm{e}}=1 \mathrm{~cm}^{-3}$ and LMC abundances ( - 1.4 to -0.4, Allen et al. 2008).

Another possibility discussed by Garnett et al. (1991) is hot $(T \lesssim 70000 \mathrm{~K}$ ) stellar ionizing continua. This looks like a plausible explanation for those cases where we had detected nebular He II $\lambda 4686$ on top of the blue bump (WR 4 and WR 5).

The last option would be photoionization caused by X-rays. The only point sources detected by Summers et al. (2004) that fall in our f.o.v. are sources 17-19. This last source appears to be associated with the Complex \#1 and thus, not related to this discussion (since no nebular He II was detected for this region). Sources 18 and 17 could, however, be associated with He II- 1 and He II-4 detections, respectively. In particular, the latter region coincides with the secondary peak of emission in the $\mathrm{H} \alpha$ image and is associated with cluster 17 of the sample catalogued by Harris et al. (2004). No satisfactory explanation was found for the cause of the ionization at He II-2 and He II-3.

\subsection{Kinematics of the ionized gas}

Slit observations in specific regions of this galaxy have demonstrated that the kinematics of the ionized gas is rather complex, with line profiles revealing asymmetric wings (e.g. Martin \& Kennicutt 1995; López-Sánchez et al. 2007). These observations usually include the bright core of the galaxy. However, they might be biased since typically the long slits only sample specific regions selected by the particular slit placement. The present data permit a 2D spatially resolved analysis of the kinematics of the ionized gas in the central area of the galaxy to be performed, thus overcoming this drawback. We based our analysis on the strongest emission lines (i.e. mainly $\mathrm{H} \alpha$, but also $\mathrm{H} \beta,[\mathrm{O}$ III] $] \lambda 5007,[\mathrm{~N} \mathrm{II}] \lambda 6584$, and [S II] $\lambda \lambda 6717,6731)$ where the high $\mathrm{S} / \mathrm{N}$ permits the line profiles to be fitted with a high degree of accuracy.

In the following, we will present the results derived from $\mathrm{H} \alpha$. Similar results were obtained from the $\mathrm{H} \beta$ and [O III] $\lambda 5007$ emission lines with differences of $|\Delta v| \lesssim 2 \mathrm{~km} \mathrm{~s}^{-1}$ in most cases and always between -5 and $5 \mathrm{~km} \mathrm{~s}^{-1}$. Results for the only emission lines with remarkable differences in the velocity maps (i.e. [N II] $\lambda 6584$ and [S II] $\lambda \lambda 6717,6731)$ will be presented, as well.

Typical examples of the different profiles for the main emission lines are shown in Fig. 16. Lines are ordered by wavelength from bluer (lower) to red (upper) within each panel. The zero point in the abscissa axis corresponds to the measured systemic velocity which is defined as the average of the velocities derived from the main emission lines for the peak of the continuum emission. We performed an independent fit for each of the brightest emission lines using MPFITEXPR (see Sect. 2.3 for details). In general, the line profiles of the individual spectra cannot be properly reproduced by a single component. A large percentage 
A. Monreal-Ibero et al.: The central region of NGC 5253 with FLAMES
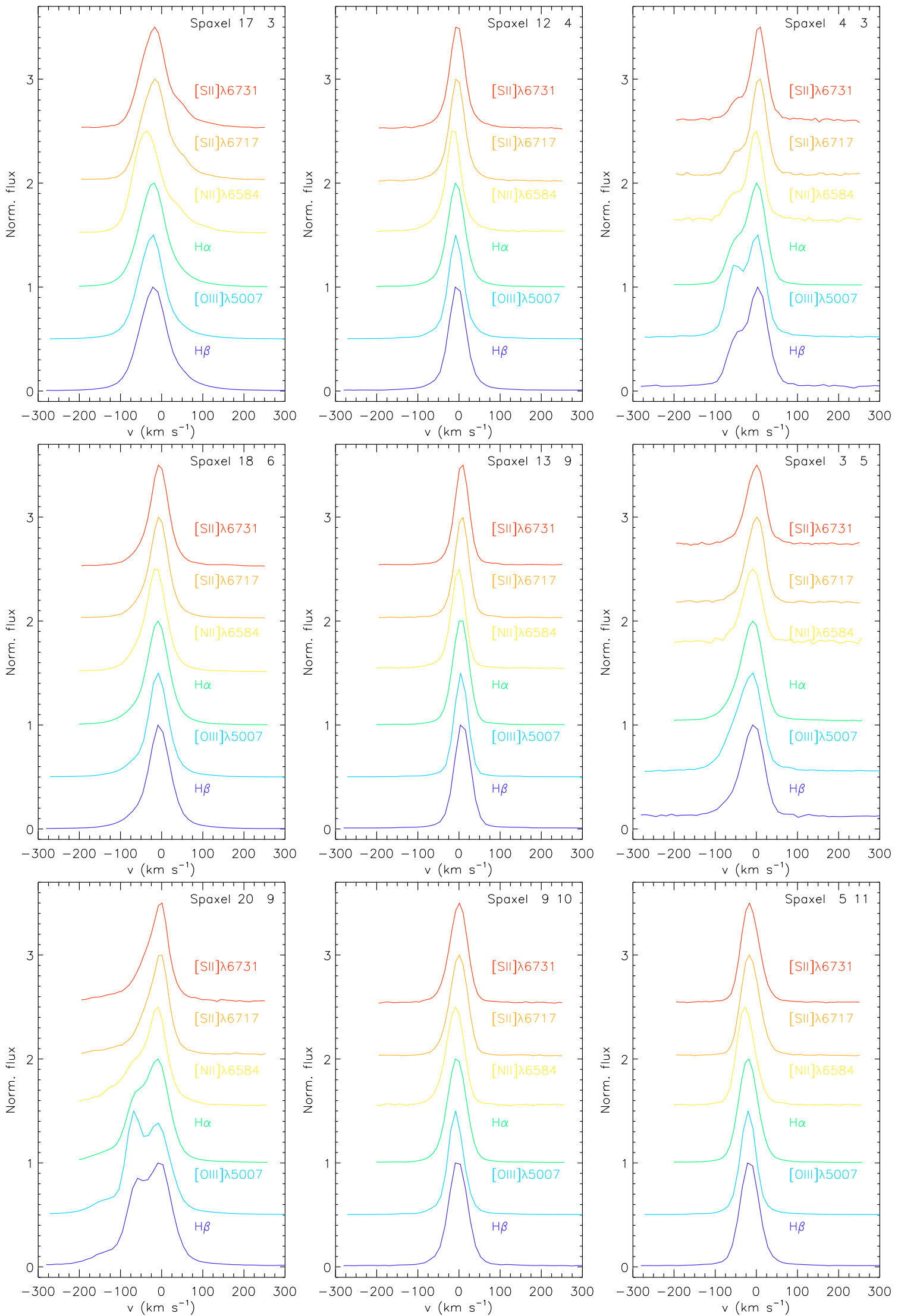

Fig. 16. Profiles of the main emission lines for representative spectra. The distribution within the FLAMES f.o.v. has been roughly retained. That is: spaxel $(1,1)$ is located at the upper right corner of the FLAMES IFU and numbering increases towards the left and bottom. The middle row contains the profiles corresponding to the positions of the peaks of continuum emission (left: Complex \#1; centre: Complex \#2; right: Complex \#3). Spectra in the left column correspond to spaxels associated with the giant $\mathrm{H}$ II region (upper: extension towards the north-west; lower: extension towards the south-east), those in the central column to areas in the centre of the FLAMES f.o.v. and those in the right column to the area associated with Complex \#3 (upper: position towards the north-west; lower: towards the south-east). 

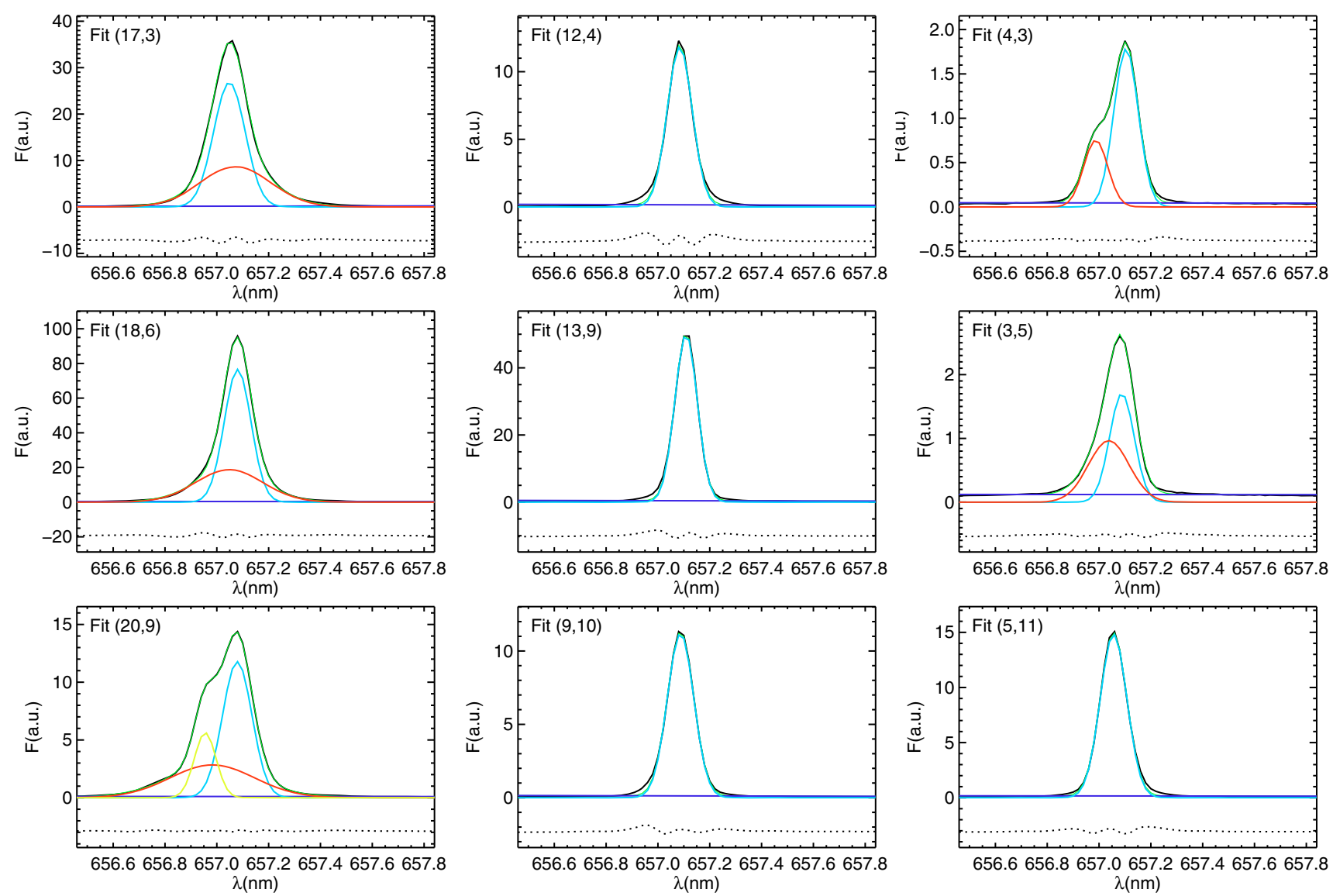

Fig. 17. Fits in $\mathrm{H} \alpha$ for the representative spectra shown in Fig. 16. Flux is in arbitrary units. The observed spectra are presented in black. The first, second and third components have been displayed in blue, red, and yellow, respectively, while the underlying continuum and total fit are in violet and green. Residuals are shown as a dotted line below the profile.

of them needed two (and even three) independent components to reproduce the observed profile reasonably well. We followed the approach of keeping the analysis as simple as possible. Thus in those cases where both fits - the one with one component and the one with several components - reproduced equally well the line profile, we gave preference to the fit with one component. Examples of these fits are shown in Fig. 17, which contains the $\mathrm{H} \alpha$ emission line together with the total fit and the individual components overplot for the spaxels shown in Fig. 16. Note how the fits for the spaxels in the central area of our f.o.v. present relatively larger residuals. These can be attributed to a low surface brightness broad extra component which would be the subject of a future work. Central wavelengths were translated into heliocentric velocity taking into account the radial velocity induced by the Earth's motion at the time of the observation which was evaluated using the IRAF task rvcorrect. Velocity dispersions were obtained from the measured FWHM after correcting for the instrumental width and thermal motions. The width of the thermal profile was derived assuming $T_{\mathrm{e}}=11650 \mathrm{~K}$ which translates into a $\sigma_{\text {ther }}=\sqrt{k T_{\mathrm{e}} / m_{\mathrm{H}}}$ of $\sim 11 \mathrm{~km} \mathrm{~s}^{-1}$ for the hydrogen lines. The measured systemic velocity was $392 \mathrm{~km} \mathrm{~s}^{-1}$. This is slightly lower than the one measured from neutral hydrogen $\left(407 \mathrm{~km} \mathrm{~s}^{-1}\right.$, Koribalski et al. 2004) according to NED.

In Fig. 18, we present the velocity fields for the three fitted components derived from the $\mathrm{H} \alpha$ emission line. We also included the velocity dispersion map for our broadest component. Corrected velocity dispersions for the two narrow components were, in general, subsonic and will not be shown here. The only exception would be an area at $\sim\left[4{ }^{\prime \prime} 0,-22^{\prime} \cdot 0\right]$. The line profiles in this area show how the narrow component presents a continuity with the two narrow components at $\sim\left[4{ }^{\prime \prime}\left(0,-1{ }^{\prime \prime} .0\right]\right.$ as if it were the result of a strong blending of these two components. However, we were not able to properly deblend these two components by means of our line fitting technique.

From Fig. 18, it is clear that the movements of the ionized gas are far from simple rotation. For the discussion we will separate the emitting area into the zone corresponding to the giant $\mathrm{H}$ II region and the rest. The zone of the giant $\mathrm{H}$ II region, occupying roughly the left part of the FLAMES field of view, shows in the upper part, line profiles that can be explained by two components while in some spaxels of the lower part a third component was required. The area of the giant $\mathrm{H}$ II region itself, which occupies an area of $\sim 120 \mathrm{pc} \times 60 \mathrm{pc}$, requires up to three components to properly reproduce the line profiles. They were named $\mathrm{C} 1, \mathrm{C} 2$ and $\mathrm{C} 3$, according to their relative fluxes. The first component (i.e. C1) accounts for the $\sim 45-68 \%$ of the flux in $\mathrm{H} \alpha$, depending on the considered spaxel. It is relatively narrow and constant, with a $\Delta v \sim 10 \mathrm{~km} \mathrm{~s}^{-1}$ over a distance of $\sim 6^{\prime \prime}$ $(\sim 110 \mathrm{pc})$ with slightly bluer velocities in the spaxels associated with the edge of the upper and lower extensions.

The second component (i.e. C2) accounts for the $\sim 27-55 \%$ of the flux in $\mathrm{H} \alpha$. It is symmetric with respect of an axis that goes through the Complex \#1 in the north-south direction. In comparison with the first component, it presents large velocity variations (i.e. $\Delta v \sim 70 \mathrm{~km} \mathrm{~s}^{-1}$ over $\sim 4$ ". 7 or $\sim 86 \mathrm{pc}$ ) and is relatively broad $\left(\sigma \sim 20-25 \mathrm{~km} \mathrm{~s}^{-1}\right)$. Low surface brightness broad components have been reported in starburst galaxies using a slit since more than a decade and have been the subject 


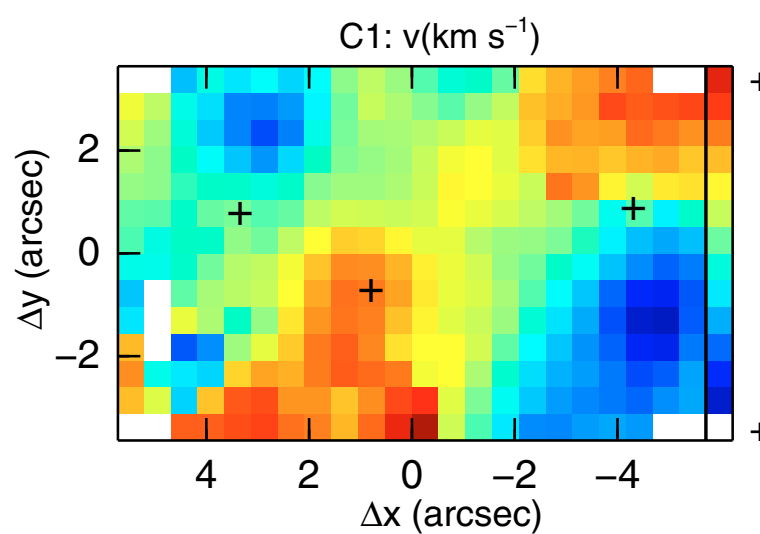

$+415$

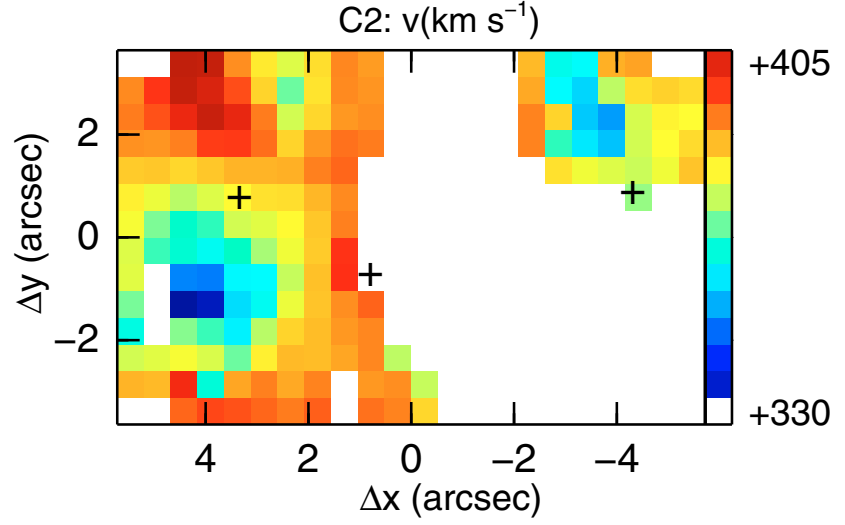

C3: $\mathrm{v}\left(\mathrm{km} \mathrm{s}^{-1}\right)$
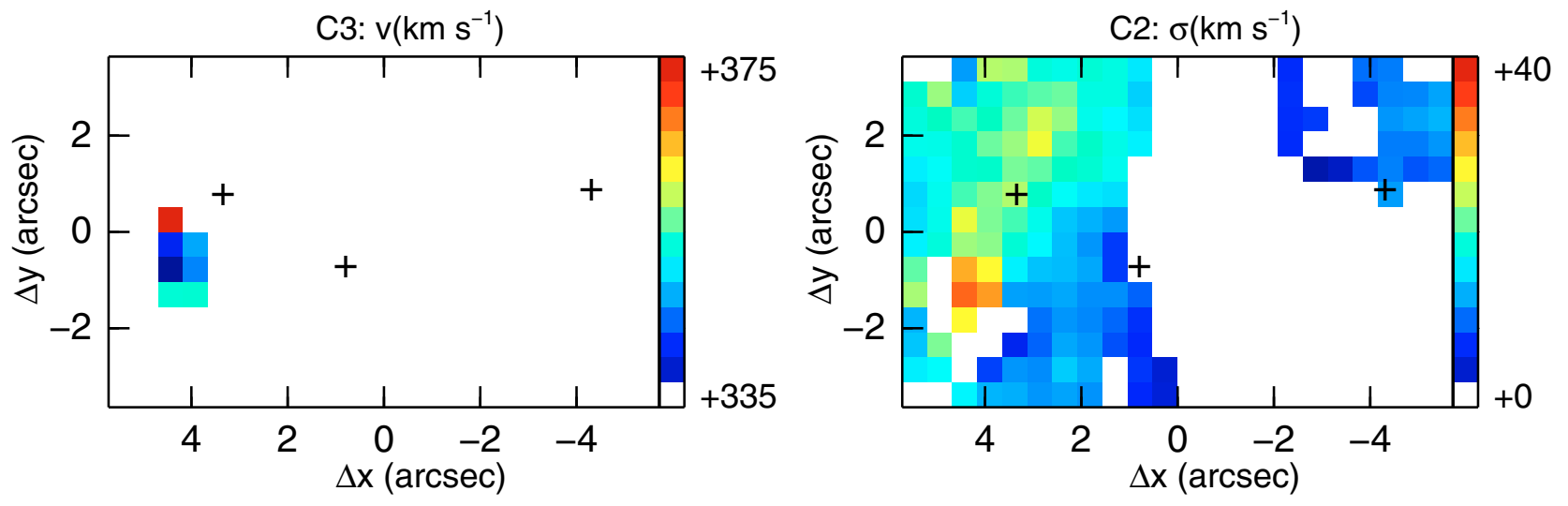

Fig. 18. Kinematic information derived from the $\mathrm{H} \alpha$ emission line. Left: velocity fields for the two narrow components. Right: velocity field (upper) and velocity dispersion map (lower) for the broad component.

of several theoretical (e.g. Tenorio-Tagle et al. 1997) and observational (e.g. Castañeda et al. 1990; González-Delgado et al. $1994)$ studies. They usually represent a small fraction $(\sim 3-20 \%)$ of the total $\mathrm{H} \alpha$ flux and have widths of $\sigma \sim 700 \mathrm{~km} \mathrm{~s}^{-1}$. Recently, 2D spectroscopic analysis of very nearby starbursts have shown how locally, the line width is somewhat smaller $\left(\sigma \sim 50-170 \mathrm{~km}^{-1}\right.$, see Westmoquette et al. 2009, and references therein). This is understood in the context of the so-called Turbulent Mixing Layers (e.g. Slavin et al. 1993). However, the high surface brightness of $\mathrm{C} 2$, together with the symmetry in the velocity field and its low widths made us to explore an alternative explanation for it (see Sect. 4.1).

The third component (i.e. C3) is present in a small area of the field of about 1.'0 diameter (i.e. $18 \mathrm{pc}$ ) and centered at about $\left[4{ }^{\prime \prime} 0,-1{ }^{\prime \prime}\right.$ 0]. C3 has a width similar to the first component (C1) but displaced $\sim 50 \mathrm{~km} \mathrm{~s}^{-1}$ towards the blue. This third component appears in a location about 1'.5 south-east to the position of the SSC complexes in the tongue-shaped extension described in Sect. 3.1. This area presents low values of extinction (see Fig. 3) and surface brightness (see Fig. 2). This third component shows indications of more extended weaker emission which was not fitted.

We pointed out before that velocity maps derived from the [N II] $\lambda 6584$ and [S II] $\lambda \lambda 6717,6731$ emission lines showed areas with important differences with respect to the one obtained from $\mathrm{H} \alpha$. This is the case for the giant H II region. Figure 19 contains the measured velocity differences for the two brighter components $(\mathrm{C} 1$ and $\mathrm{C} 2)$ in the subset of the field corresponding to the giant $\mathrm{H}$ II region while Fig. 20 presents examples of the independent fits for $\mathrm{H} \alpha$, [N II] $\lambda 6584$, and [S II] $\lambda 6717$. Differences exist for both, the narrow (i.e. C1) and broad (i.e. C2), components in $[\mathrm{N} \mathrm{II}] \lambda 6584$ and are relatively symmetric with respect to the peak of continuum emission Complex \#1, but with opposite sign and slightly different directions $\left(\mathrm{PA} \sim 50^{\circ}\right.$ and $\sim 30^{\circ}$ for $\mathrm{C} 1$ and $\mathrm{C} 2$ respectively). Also, the range of velocity differences, $\left(v_{\mathrm{H} \alpha}-v_{[\mathrm{NII}]}\right)$ is larger for $\mathrm{C} 2$ than for $\mathrm{C} 1\left(\sim 70 \mathrm{~km} \mathrm{~s}^{-1}\right.$ and $\sim 30 \mathrm{~km} \mathrm{~s}^{-1}$, respectively). As illustrated in the right hand map of Fig. 19, the broad component for the [S II] $\lambda \lambda 6717,6731$ emission lines also shows velocity differences with similar range $\left(v_{\mathrm{H} \alpha}-v_{[\mathrm{SII}]} \sim 60 \mathrm{~km} \mathrm{~s}^{-1}\right)$, orientation and sign as in the case of the $[\mathrm{N}$ II $] \lambda 6584$ emission line. For the $\mathrm{C} 1$ component, no significant differences were found. Measured differences in $\mathrm{C} 3$, with a mean and standard deviation of $-6 \pm 9 \mathrm{~km} \mathrm{~s}^{-1}$ and $5 \pm 4 \mathrm{~km} \mathrm{~s}^{-1}$ for $v_{\mathrm{H} \alpha}-v_{[\mathrm{NII}]}$ and $v_{\mathrm{H} \alpha}-v_{[\mathrm{SII}]}$ respectively, do not appear to be significant. However, since C3 was only detected in seven spaxels (see Fig. 18, bottom left panel) this result has to be treated with caution.

Similar offsets has been detected in galactic $\mathrm{H}$ II regions like Orion (García-Díaz et al. 2008), but to our knowledge, this is the first time that maps with such offsets in velocity for different emission lines in starbursts are presented. This can partially be caused by the fact that 2D-kinematic analysis of starbursts, from dwarfs (e.g. García-Lorenzo et al. 2008) to more extreme events like LIRGs (e.g. Alonso-Herrero et al. 2009), are usually based on fitting techniques that impose restrictions between the $\mathrm{H} \alpha$ and $[\mathrm{N}$ II $] \lambda 6584$ central wavelengths and thus, preventing from detecting such offsets. An example of work where the main emission lines are fitted independently is presented by Westmoquette et al. (2007). However, since they only analyzed the kinematic for $\mathrm{H} \alpha$, it is not possible to assess if they found different kinematics for the other emission lines. There are however, some works that offer examples of offsets of this kind using a slit. In particular López-Sánchez et al. (2007) report also an offset between the $\left[\mathrm{N}\right.$ II] $\lambda 6584$ and the $\mathrm{H} \alpha$ emission line of $\sim 10 \mathrm{~km} \mathrm{~s}^{-1}$, 

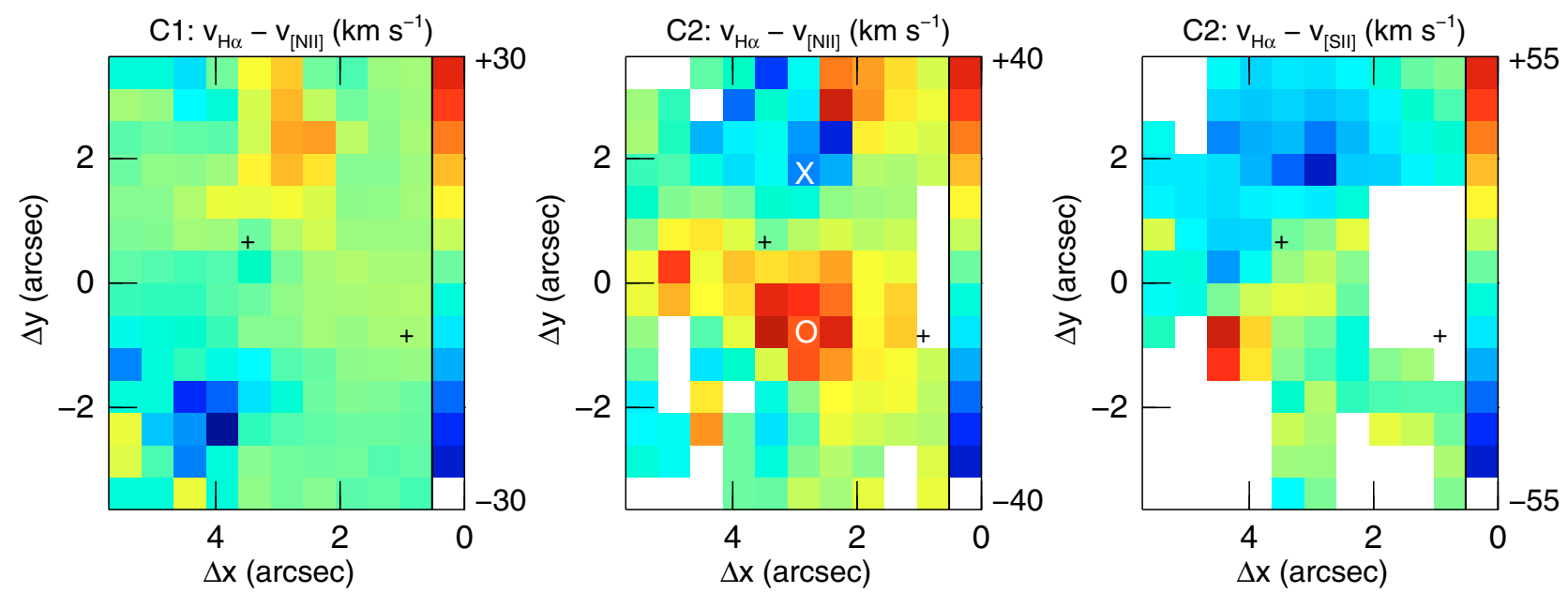

Fig. 19. Difference between the velocity fields derived from the $\mathrm{H} \alpha$ and the $[\mathrm{N}$ II $] \lambda 6584$ emission lines for the narrow (left) and broad (center) components as well as between those derived from the $\mathrm{H} \alpha$ and [S II] $\lambda \lambda 6717,6731$ emission lines for the broad component (right). Only the section of the FLAMES field of view associated with the giant $\mathrm{H}$ II region is shown. The "X" and the "O" symbols in the central panel show the position of the spaxels $(17,4)$ and $(17,9)$, repectively (see line fitting in Fig. 20).
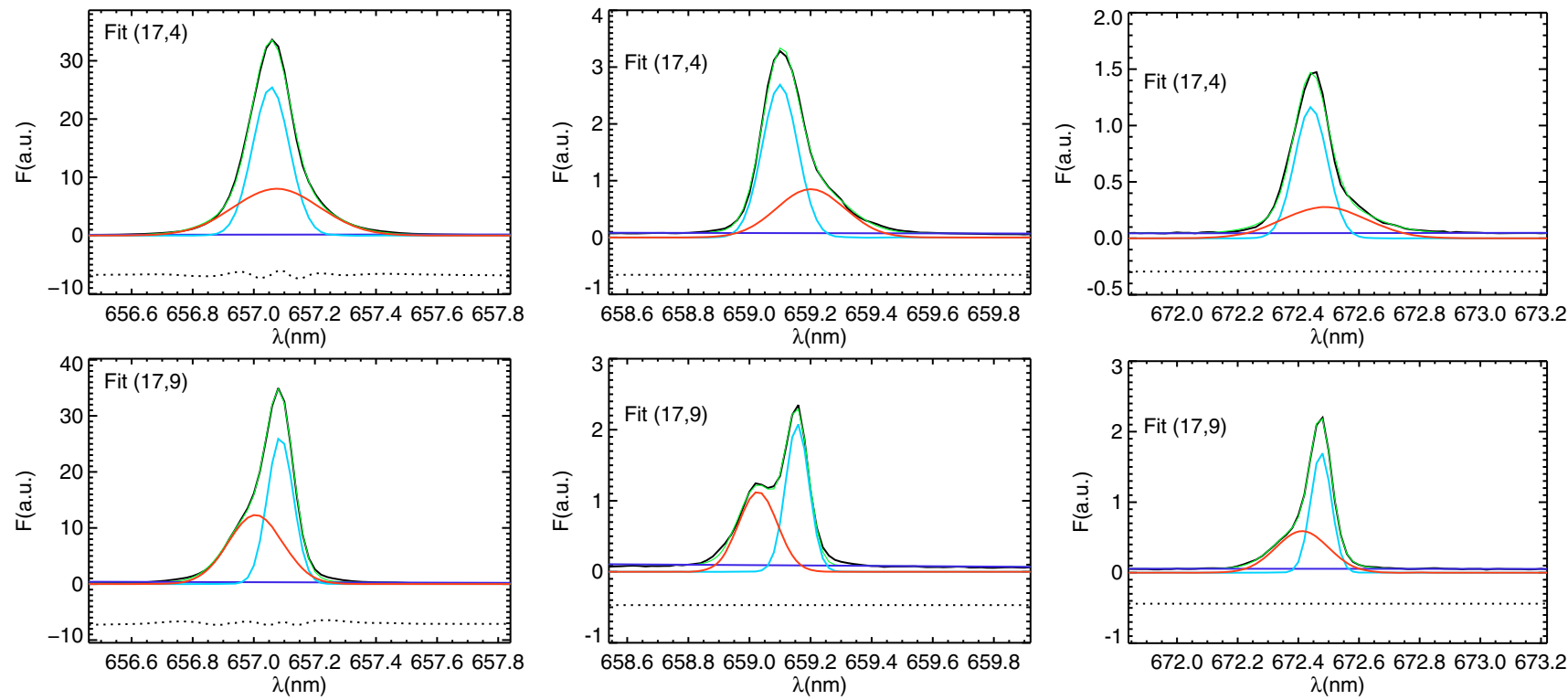

Fig. 20. Fits in $\mathrm{H} \alpha$ (left), [N II] 66584 (middle), and, [S II] 66717 (right) for two representative spaxels showing offsets in velocity for the different emission lines 16. Flux is in arbitrary units. The observed spectra are presented in black. The first and second components have been displayed in blue and red, respectively, while the underlying continuum and total fit are in violet and green. Residuals are shown as a dotted line below the profile.

similar to what we have measured for $\mathrm{C} 1$ in the upper part of our f.o.v.

The second region of interest is located in the right (south-west) part of the FLAMES field of view. Emission there shows narrow lines with velocity dispersion dominated by the thermal width. In some areas (the north-east corner in Fig. 18), two narrow lines were needed to better reproduce the line profile. The primary component (i.e. C1) shows a symmetric velocity pattern with respect to the twin clusters associated with the peak of emission \#3. A velocity gradient in the north-west to south-east direction is clear with a $\Delta v \sim 40 \mathrm{~km}^{-1}$ over about 4 ". $^{\prime} 0$ (linear scale of $\sim 75 \mathrm{pc}$ ). The secondary component traces a shell blue-shifted $\sim 40 \mathrm{~km} \mathrm{~s}^{-1}$ in the western corner (see Fig. 18, upper right panel). Note that $\mathrm{C} 2$, although relatively narrow, is a bit broader than the thermal width (Fig. 18, bottom right panel). This component accounts for $\sim 30-50 \%$ of the $\mathrm{H} \alpha$ flux in this area. No significant differences in the velocity fields and the velocity dispersion maps for the main emission lines have been found in this area.

\section{Discussion}

\subsection{The giant $\mathrm{H} / \mathrm{l}$ region}

The most interesting area of NGC 5253 in the present data covers the left (north-east) part of the FLAMES field of view. In previous sections we have seen that this area is occupied by a giant $\mathrm{H}$ II region which: i) harbors two very massive and young SSCs at its centre (i.e. Complex \#1); ii) presents high levels of extinction, being larger in the upper part of the f.o.v.; iii) has high electron densities as traced by both the sulphur and the argon line ratios, and again are also larger in the upper part of 


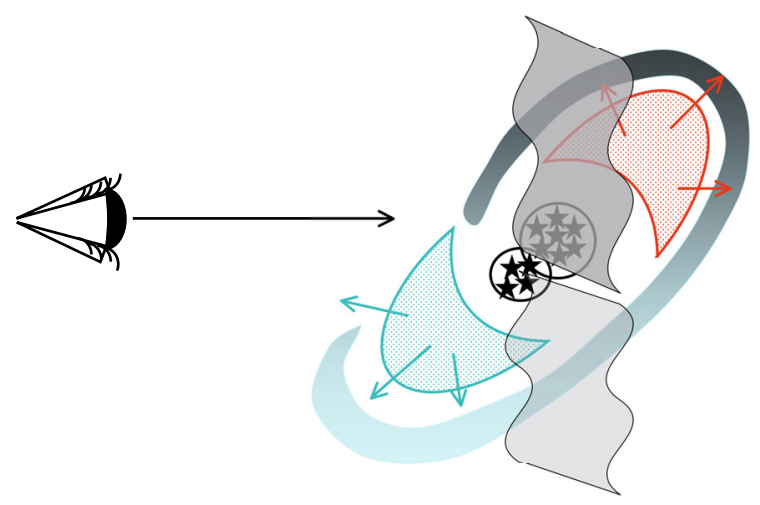

Fig. 21. Sketch showing the different elements associated with the area of the giant HII region. The two massive SSCs (stars in the circles) expel material (red and blue half moons) which encounter quiescent gas (grey shell). Regions of dust extinction are represented by the two wavy sheets.

the f.o.v.; iv) presents an excess in the $[\mathrm{N} \mathrm{II}] \lambda 6584 / \mathrm{H} \alpha$ line ratio with respect to $[\mathrm{S} \mathrm{II}] \lambda \lambda 6717,6731 / \mathrm{H} \alpha$ which, if interpreted as $\mathrm{N}$-enrichment, indicates an outward gradient of extra nitrogen from a point at $\sim 0 .{ }^{\prime} 5-1$.' 0 towards the north-west of the peak of continuum emission at Complex \#1; v) presents W-R features, implying a young age for the harboured stellar population; vi) displays complex kinematics, not coincident in all the emission lines, that require a minimum of three components to reproduce the line profiles. How does all this evidence fit together into a coherent picture?

In Fig. 21, we sketch a plausible scenario compatible with all these results. Here, the broad component (C2) would trace an outflow created by the two SSCs at Complex \#1 while the two narrow components ( $\mathrm{C} 1$ and $\mathrm{C} 3$ ) would be caused by a shell of previously existing quiescent gas that has been reached by the ionization front. Different grades of grey in Fig. 21 in the shell represent the different densities observed in the upper and lower part of the FLAMES f.o.v., while two wavy sheets in two grades of grey have been used to represent the differences in extinction between these two halves. Due to this extinction distribution, in the upper (i.e. north-western) half of the $\mathrm{H}$ II region, the observer cannot see the further part of the shell, while in the lower (i.e. south-eastern) half both parts are visible and are detected as a single broader component when approaching the vertex of the oval.

As in Sect. 3.3, we formed [S II] $\lambda 6717 /[\mathrm{S} \mathrm{II}] \lambda 6731$ maps for the individual kinematic components and measured the mean and standard deviation for the sulphur line ratio in two $4 \times 4$ spaxel squares sampling the upper and lower part of the giant $\mathrm{H}$ II region. Although the standard deviations are large $(\sim 0.09)$, results support this sketch. While the broad component presented similar line ratios in both areas $(\sim 1.07$ implying densities of $\sim 470 \mathrm{~cm}^{-3}$ ), the narrow component presented somewhat lower line ratios in the upper part than in the lower one $(\sim 1.11$ vs. $\sim 1.25)$ implying densities for the shell of $\sim 390 \mathrm{~cm}^{-3}$ and $\sim 180 \mathrm{~cm}^{-3}$, respectively. Also, we created (noisier) [N II] $\lambda 6584 / \mathrm{H} \alpha$, [S II] $] \lambda 6717,6731 / \mathrm{H} \alpha$, and $[\mathrm{O}$ III $] \lambda 5007 / \mathrm{H} \beta$ line maps and compared the relation between [S II] $\lambda \lambda 6717,6731 / \mathrm{H} \alpha$ and $[\mathrm{N} \mathrm{II}] \lambda 6584 / \mathrm{H} \alpha$ for the individual components. The three fitted components present extra nitrogen in an area coinciding with the one derived from one-gaussian fitting. This differs from the findings for Mrk 996, a galaxy with several kinematically distinct components where only the broad one presented $\mathrm{N}$-enrichment, with an abundance 20 times larger than the one for the narrow component (see James et al. 2009). Still, in NGC 5253, the N-enrichment in the broad component is larger than in the narrow one by a factor of $\sim 1.7$ which is consistent with the scenario sketched above. Moreover, while the $[\mathrm{O} \mathrm{III}] \lambda 5007 / \mathrm{H} \beta$ maps are relatively similar for both components ${ }^{6}$ (not shown), those associated to [N II] $\lambda 6584 / \mathrm{H} \alpha$ and specially to (the more shock sensitive) $[\mathrm{S} \mathrm{II}] \lambda \lambda 6717,6731 / \mathrm{H} \alpha$ line ratio display a relatively different ionization degree with larger values for $\mathrm{C} 1$ than for $\mathrm{C} 2$. This is also consistent with the presented scenario since a larger contribution due to shocks is expected in the area where the outflowing material encounters the pre-existent gas.

An interesting result of the previous section was the offsets derived for the velocities of the different species, in particular nitrogen and sulphur. To our knowledge, this is the first time that maps showing this kind of offsets are reported in an starburst galaxy. Similar phenomena have already been reported in much closer regions of star formation. For example, observations in the Galactic Orion Nebula, a much less extreme event in terms of star formation, show how $\mathrm{H} \alpha$ and [O III] $] 25007$ display similar velocities while [N II] $\lambda 6584$ and [S II] $\lambda \lambda 6717,6731$ are shifted by $\sim 4-5 \mathrm{~km} \mathrm{~s}^{-1}$ (García-Díaz et al. 2008), an order of magnitude smaller than the shifts found for NGC 5253. Also, self-consistent dynamic models of steady ionization fronts point towards the detection of such differences (Henney et al. 2005). In the context of the scenario sketched in Fig. 21, the offsets in C2 would fit if $[\mathrm{N} \mathrm{II}] \lambda 6584$ (and [S II] $\lambda \lambda 6717,6731$ ) traced the outer parts in an outflow which has a Hubble flow (i.e. velocity proportional to radius).

Finally, an estimate of the time scales associated with the pollution process can be determined by using the velocity for the outflow derived in Sect. 3.7. Assuming that this traces the velocity of nitrogen contamination of the ISM, the detected pollution extending up to distances of $\sim 60$ pc took place over only $\sim 1.3-1.7 \mathrm{Myr}$. This supports the idea that the nitrogen dilution is a relatively fast process and is consistent with the shortage of observed systems presenting this kind of chemical inhomogeneity.

\subsection{The area associated with the older stellar clusters}

The rightmost (south-west) part of the FLAMES f.o.v. presents a different picture. We have seen that this region: i) is associated with two relatively old ( $\sim 70$ and $\sim 110 \mathrm{Myr})$ and massive (3 and $7 \times 10^{4} M_{\odot}$ ) clusters (Harris et al. 2004); ii) presents moderate levels of extinction, being higher in the lower part of the FLAMES field of view; iii) has very low $N_{\mathrm{e}}$ and lower than $100 \mathrm{~cm}^{-3}$ in the upper corner; iv) the $\mathrm{H} \alpha$ surface brightness is very low (i.e. one and two orders of magnitude smaller than in the giant $\mathrm{H}$ II region for the upper and lower portions respectively); v) displays two distinct kinematic components in the upper part of the f.o.v.. Noteworthy is that two supernova remnant candidates have been detected in the area (Labrie \& Pritchet 2006). One of them (S001) appears very close in projection to the massive clusters at $\sim\left[-5^{\prime \prime} 0,11^{\prime \prime} 0\right]$. The second one $(\mathrm{S} 002)$ is located, just outside of the FLAMES f.o.v., at the right upper corner.

The first question to consider is if any of the kinematic components is related to the supernova remnant candidates. However, our measured velocity dispersions are much more lower than expansion velocities of typical supernova remnants (i.e. NGC 1952, $1450 \mathrm{~km} \mathrm{~s}^{-1}$ Osterbrock \& Ferland 2006).

\footnotetext{
${ }^{6} \mathrm{C} 3$ is not considered here, since the so-called map would be associated to $\leq 7$ spaxels, depending on the line ratio.
} 
Table 5. Integrated line ratios for the rightmost (south-west) part of the FLAMES f.o.v.

\begin{tabular}{cccc}
\hline \hline Component & $\log ([\mathrm{O} \mathrm{III}] / \mathrm{H} \beta)$ & $\log ([\mathrm{N} \mathrm{II}] / \mathrm{H} \alpha)$ & $\log ([\mathrm{S} \mathrm{II}] / \mathrm{H} \alpha)$ \\
\hline Upper Blue & 0.69 & -1.20 & -0.85 \\
Upper Red & 0.52 & -1.09 & -0.67 \\
Lower & 0.54 & -1.09 & -0.75 \\
${\text { NGC } 1952^{a}}$ & 0.92 & 0.15 & 0.18 \\
\hline
\end{tabular}

Notes. ${ }^{(a)}$ From Osterbrock \& Ferland (2006).

Moreover, we created two integrated spectra for the upper and the lower part of the area. Line ratio for both components of the upper part and for the lower part were relatively similar (i.e. within $\sim 0.1$ dex, see Table 5) and much lower than those expected for a supernova remnant (e.g. NGC 1952, Osterbrock $\&$ Ferland 2006). Thus, supernova remnants are not obviously the cause of the observed kinematics and physical properties of the ionized gas in this region.

Instead, given that all the three components present similar line ratios - consistent with ionization caused by stars - and similar line widths, a more plausible scenario would be that where all the components are part of a common picture. Given the age of the clusters, and the velocity differences between the three components, this area can be viewed as a snapshot of a more evolved version of what is happening in the left part of the FLAMES f.o.v. The clusters have managed to clear out their environment. Only a broken shell made out of previously quiescent gas remains ionized by the remaining hot stars and moving away from the clusters with little evidence for high velocity outflow. Figure 22 presents a sketch of the different elements associated with this area.

\section{Summary}

We present a thorough study of the ionized gas and its relation with the stellar population of NGC 5253 by mapping the central $212 \mathrm{pc} \times 134 \mathrm{pc}$ in a continuous and unbiased manner using the ARGUS IFU unit of FLAMES. The analysis of the data have yield the following results.

1. We obtained a 2D detailed map for the extinction suffered by the ionized gas, finding an offset of $\sim 0$.' 5 between the peak of the optical continuum and the extinction peak, in agreement with findings in the infrared.

2. We compared the extinction suffered by gas and stars by defining ad hoc broad-band colours. We have shown the importance of using line-free filters when performing this comparison and found that stars suffer less extinction than the ionized gas by a factor $\sim 0.33$, similar to the findings in other starburst galaxies.

3. We derived $N_{\mathrm{e}}$ sensitive line ratio maps. The one involving the sulphur lines shows a gradient from $790 \mathrm{~cm}^{-3}$ at the peak of emission in the giant $\mathrm{HII}$ region described by Calzetti et al. (1997) outwards. The argon line ratio is only detected in the area associated with the giant $\mathrm{H}$ II region and traces the highest density $\left(\sim 4200-6200 \mathrm{~cm}^{-3}\right)$ regions.

4. We studied the ionization structure by means of the maps of line ratios involved in the BPT diagrams. The spatial distribution of the [S II] $\lambda \lambda 6717,6731 / \mathrm{H} \alpha$ and [O III] $\lambda 5007 / \mathrm{H} \beta$ line ratios follows that for the flux distribution of the ionized gas. On the contrary, the [N II] $\lambda 6584 / \mathrm{H} \alpha$ map shows a completely different structure.

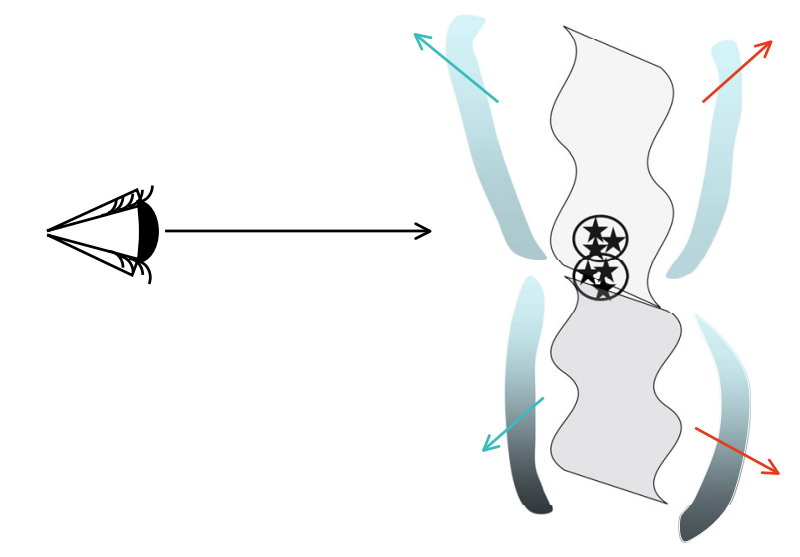

Fig. 22. Sketch showing the different elements associated with the right part of the FLAMES field of view. The two massive SSCs (stars in the circles) have already expelled their material and are seen as relatively quiescent ionized shells (shown in grey). Differences in extinction are represented by two wavy sheets.

5. We evaluated the possible ionization mechanisms through the position of these line ratios in the diagnostic diagrams and comparing with the predictions of models. All our line ratios are compatible with photoionization caused by stars. The [S II] $\lambda \lambda 6717,6731 / \mathrm{H} \alpha$ indicated a somewhat higher ionization degree that might be evidence of some contribution of shocks to the measured line ratios. Part of the data in the diagram involving the $[\mathrm{N} \mathrm{II}] \lambda 6584 / \mathrm{H} \alpha$ line ratio are distributed in a distinct cloud. This can be explained within the local $\mathrm{N}$-enrichment scenario proposed for this galaxy.

6. We delimited very precisely the area presenting local $\mathrm{N}$-enrichment. It occupies the whole giant $\mathrm{H}$ II region, including the two extensions towards the upper and lower part of the FLAMES field of view, peaking at $\sim 1$."5 from the peak of emission in the continuum and almost coincident (i.e. at $\sim 20 \mathrm{pc}$ ) with the peak of extinction.

7. We located the areas that could contain Wolf-Rayet stars by looking for the blue bump. We confirmed the existence of W-R stars associated with the nucleus and the brightest cluster in the ultraviolet. W-R stars are distributed in a wider area than the one presenting N-enrichment and in a more irregular manner. We were able to identified one (or more) clusters with ages compatible with the existence of W-R stars in all but one (i.e. W-R 5) of our delineated regions with a W-R signature.

8. If the scenario of $N$-enrichment caused by $W$-R stars turns out to be applicable, only the W-R detected at the core (Complex \#1), and perhaps in the two extensions of the Giant H II region, can be considered the cause of the local $\mathrm{N}$-enrichment, according to the correlation of the spatial distribution of $\mathrm{W}-\mathrm{R}$ features and $\mathrm{N}$-enrichment.

9. We measured the $\mathrm{He}^{+}$and $\mathrm{He}^{++}$abundances. $\mathrm{He}^{+} / \mathrm{H}^{+}$is 0.08-0.09 in most of our field of view except for an area of $\sim 2^{\prime \prime} \times 3^{\prime \prime}$ in the upper right corner, far away from the main ionization source. We detected the nebular He II $\lambda 4686$ emission line in areas not coincident, in general, with those presenting W-R features, nor with the one presenting $\mathrm{N}$-enrichment. Abundances in He II were always $\$ 0.0005$. Given the depth and unbiased mapping of the present data, we can exclude the possibility of further detections of larger quantities of $\mathrm{He}^{++}$based on optical observations in the nuclear region of NGC 5253. This result is difficult to reconcile with the scenario of $\mathrm{N}$-enrichment caused by 
$W$ - $R$ stars and favours a suggestion where the N-enrichment arises during the late $\mathrm{O}$-star wind phase.

10. We studied the kinematics of the ionized gas by using velocity fields and velocity dispersion maps for the main emission lines. We needed up to three components to properly reproduce the line profiles. In particular, one of the components associated with the Giant H II region presents supersonic widths and [N II] $\lambda 6584$ and [S II] $\lambda \lambda 6717,6731$ emission lines shifted up to $40 \mathrm{~km} \mathrm{~s}^{-1}$ with respect to $\mathrm{H} \alpha$. Also, one of the narrow components shows velocity offsets in the [N II] $\lambda 6584$ line of up to $20 \mathrm{~km} \mathrm{~s}^{-1}$. This is the first time that maps providing such offsets for a starburst galaxy have been presented.

11. We provide a scenario for the event occurring at the Giant $\mathrm{H}$ II region. The two SSCs are producing an outflow that encounters previously existing quiescent gas. The scenario is consistent with the measured extinction structure, electron densities and kinematics.

12. We explain the different elements in the right (south-west) part of the FLAMES field of view as a more evolved stage of a similar scenario where the clusters have now cleared their local environment. This is supported by the low electron densities and $\mathrm{H} \alpha$ surface brightness as well as the kinematics in this area.

Acknowledgements. We thank Peter Weilbacher for his help in the initial stages of this project as well as Christer Sandin for useful discussions about differential atmospherical refraction correction. We also thank the anonymous referee for his/her careful and detailed review of the manuscript. Based on observations carried out at the European Southern Observatory, Paranal (Chile), programme 078.B-0043(A). This paper uses the plotting package jmaplot, developed by Jesús Maíz-Apellániz, http://dae45.iaa.csic.es:8080/ $\sim$ jmaiz/software. This research made use of the NASA/IPAC Extragalactic Database (NED), which is operated by the Jet Propulsion Laboratory, California Institute of Technology, under contract with the National Aeronautics and Space Administration. A.M.I. is supported by the Spanish Ministry of Science and Innovation (MICINN) under the program "Specialization in International Organisations", Ref. ES2006-0003. This work has been partially funded by the Spanish PNAYA, projects ESTALLIDOS (grants AYA2007-7765-C01 and C02) and CSD2006 - 00070 "1st Science with GTC" from the CONSOLIDER 2010 programme of the Spanish MICINN.

\section{References}

Allen, M. G., Groves, B. A., Dopita, M. A., Sutherland, R. S., \& Kewley, L. J. 2008, ApJS, 178, 20

Alonso-Herrero, A., Rieke, G. H., Rieke, M. J., \& Scoville, N. Z. 2002, AJ, 124, 166

Alonso-Herrero, A., Takagi, T., Baker, A. J., et al. 2004, ApJ, 612, 222

Alonso-Herrero, A., García-Marín, M., Monreal-Ibero, A., et al. 2009, A\&A, 506, 1541

Asplund, M., Grevesse, N., Sauval, A. J., Allende Prieto, C., \& Kiselman, D. 2004, A\&A, 417, 751

Baldwin, J. A., Phillips, M. M., \& Terlevich, R. 1981, PASP, 93, 5

Bordalo, V., Plana, H., \& Telles, E. 2009, ApJ, 696, 1668

Brinchmann, J., Kunth, D., \& Durret, F. 2008, A\&A, 485, 657

Calzetti, D., Kinney, A. L., \& Storchi-Bergmann, T. 1994, ApJ, 429, 582

Calzetti, D., Meurer, G. R., Bohlin, R. C., et al. 1997, AJ, 114, 1834

Calzetti, D., Harris, J., Gallagher, III, J. S., et al. 2004, AJ, 127, 1405

Campbell, A., Terlevich, R., \& Melnick, J. 1986, MNRAS, 223, 811

Cardelli, J. A., Clayton, G. C., \& Mathis, J. S. 1989, ApJ, 345, 245

Castañeda, H. O., Vílchez, J. M., \& Copetti, M. V. F. 1990, ApJ, 365, 164

Conti, P. S. 1976, Memoires of the Société Royale des Sciences de Liège, 9, 193

Conti, P. S., Crowther, P. A., \& Leitherer, C. 2008, From Luminous Hot Stars to Starburst Galaxies, ed. P. S. Conti, P. A. Crowther, \& C. Leitherer (Cambridge University Press)

Cresci, G., Vanzi, L., \& Sauvage, M. 2005, A\&A, 433, 447

Dopita, M. A., Fischera, J., Sutherland, R. S., et al. 2006, ApJS, 167, 177

Esteban, C., Peimbert, M., Torres-Peimbert, S., \& Rodríguez, M. 2002, ApJ, 581, 241

Fluks, M. A., Plez, B., The, P. S., et al. 1994, A\&AS, 105, 311
García-Díaz, M. T., Henney, W. J., López, J. A., \& Doi, T. 2008, Rev. Mex. Astron. Astrofís., 44, 181

García-Lorenzo, B., Cairós, L. M., Caon, N., Monreal-Ibero, A., \& Kehrig, C. 2008, ApJ, 677, 201

Garnett, D. R., Kennicutt, Jr., R. C., Chu, Y., \& Skillman, E. D. 1991, ApJ, 373, 458

González-Delgado, R. M., Pérez, E., Tenorio-Tagle, G., et al. 1994, ApJ, 437, 239

González-Riestra, R., Rego, M., \& Zamorano, J. 1987, A\&A, 186, 64

Haro, G. 1956, AJ, 61, 178

Harris, J., Calzetti, D., Gallagher, III, J. S., Smith, D. A., \& Conselice, C. J. 2004, ApJ, 603, 503

Henney, W. J., Arthur, S. J., Williams, R. J. R., \& Ferland, G. J. 2005, ApJ, 621, 328

Holovatyy, V. V., \& Melekh, B. Y. 2002, Astron. Rep., 46, 779

Hora, J. L., Latter, W. B., \& Deutsch, L. K. 1999, ApJS, 124, 195

Izotov, Y. I., Schaerer, D., Blecha, A., et al. 2006, A\&A, 459, 71

James, B. L., Tsamis, Y. G., Barlow, M. J., et al. 2009, MNRAS, 398, 2

Karachentsev, I. D., Tully, R. B., Dolphin, A., et al. 2007, AJ, 133, 504

Kauffmann, G., Heckman, T. M., Tremonti, C., et al. 2003, MNRAS, 346, 1055

Kehrig, C., Vílchez, J. M., Sánchez, S. F., et al. 2008, A\&A, 477, 813

Kennicutt, Jr., R. C. 1992, ApJS, 79, 255

Kewley, L. J., \& Dopita, M. A. 2002, ApJS, 142, 35

Kewley, L. J., Dopita, M. A., Sutherland, R. S., Heisler, C. A., \& Trevena, J. 2001, ApJ, 556, 121

Kobulnicky, H. A., \& Skillman, E. D. 2008, AJ, 135, 527

Kobulnicky, H. A., Skillman, E. D., Roy, J.-R., Walsh, J. R., \& Rosa, M. R. 1997, ApJ, 477, 679

Kobulnicky, H. A., Kennicutt, Jr., R. C., \& Pizagno, J. L. 1999, ApJ, 514, 544

Koribalski, B. S., Staveley-Smith, L., Kilborn, V. A., et al. 2004, AJ, 128, 16

Kunth, D., \& Östlin, G. 2000, A\&A Rev., 10, 1

Labrie, K., \& Pritchet, C. J. 2006, ApJS, 166, 188

Lagos, P., Telles, E., Muñoz-Tuñón, C., et al. 2009, AJ, 137, 5068

Leitherer, C., Schaerer, D., Goldader, J. D., et al. 1999, ApJS, 123, 3

López-Sánchez, Á. R., Esteban, C., García-Rojas, J., Peimbert, M., \& Rodríguez, M. 2007, ApJ, 656, 168

Markwardt, C. B. 2009, in ASP Conf. Ser., ed. D. A. Bohlender, D. Durand, \& P. Dowler, 411, 251

Martin, C. L. 1998, ApJ, 506, 222

Martin, C. L., \& Kennicutt, Jr., R. C. 1995, ApJ, 447, 171

Martín-Hernández, N. L., Schaerer, D., \& Sauvage, M. 2005, A\&A, 429, 449

Osterbrock, D. E., \& Ferland, G. J. 2006, Astrophysics of gaseous nebulae and active galactic nuclei, ed. D. E. Osterbrock, \& G. J. Ferland

Pagel, B. E. J., Simonson, E. A., Terlevich, R. J., \& Edmunds, M. G. 1992, MNRAS, 255, 325

Pasquini, L., Ávila, G., Blecha, A., et al. 2002, The Messenger, 110, 1

Pérez-Montero, E., \& Contini, T. 2009, MNRAS, 398, 949

Rieke, G. H., \& Lebofsky, M. J. 1985, ApJ, 288, 618

Sakai, S., Ferrarese, L., Kennicutt, Jr., R. C., \& Saha, A. 2004, ApJ, 608, 42

Sanders, D. B., Mazzarella, J. M., Kim, D.-C., Surace, J. A., \& Soifer, B. T. 2003, AJ, 126, 1607

Schaerer, D. 1996, ApJ, 467, L17

Schaerer, D., Contini, T., Kunth, D., \& Meynet, G. 1997, ApJ, 481, L75

Schaerer, D., Contini, T., \& Pindao, M. 1999, A\&AS, 136, 35

Schlegel, D. J., Finkbeiner, D. P., \& Davis, M. 1998, ApJ, 500, 525

Shaw, R. A., \& Dufour, R. J. 1995, PASP, 107, 896

Slavin, J. D., Shull, J. M., \& Begelman, M. C. 1993, ApJ, 407, 83

Stasińska, G., Cid Fernandes, R., Mateus, A., Sodré, L., \& Asari, N. V. 2006, MNRAS, 371, 972

Strickland, D. K., \& Stevens, I. R. 1999, MNRAS, 306, 43

Summers, L. K., Stevens, I. R., Strickland, D. K., \& Heckman, T. M. 2004, MNRAS, 351, 1

Taylor, V. A., Jansen, R. A., Windhorst, R. A., Odewahn, S. C., \& Hibbard, J. E. 2005, ApJ, 630, 784

Tenorio-Tagle, G., Munoz-Tunon, C., Perez, E., \& Melnick, J. 1997, ApJ, 490, L179

Tsamis, Y. G., Barlow, M. J., Liu, X., Danziger, I. J., \& Storey, P. J. 2003, MNRAS, 345, 186

Turner, J. L., Beck, S. C., \& Ho, P. T. P. 2000, ApJ, 532, L109

Turner, J. L., Beck, S. C., Crosthwaite, L. P., et al. 2003, Nature, 423, 621

van den Bergh, S. 1980, PASP, 92, 122

Veilleux, S., \& Osterbrock, D. E. 1987, ApJS, 63, 295

Vílchez, J. M., \& Iglesias-Páramo, J. 1998, ApJ, 508, 248

Walsh, J. R., \& Roy, J.-R. 1989, MNRAS, 239, 297

Wang, W., Liu, X., Zhang, Y., \& Barlow, M. J. 2004, A\&A, 427, 873

Westmoquette, M. S., Smith, L. J., Gallagher, J. S., \& Exter, K. M. 2007, MNRAS, 381, 913

Westmoquette, M. S., Gallagher, J. S., Smith, L. J., et al. 2009, ApJ, 706, 1571 